Semiclassical description of collisionally induced rainbow satellites: a model study

This article has been downloaded from IOPscience. Please scroll down to see the full text article.

2010 J. Phys. B: At. Mol. Opt. Phys. 43215210

(http://iopscience.iop.org/0953-4075/43/21/215210)

View the table of contents for this issue, or go to the journal homepage for more

Download details:

IP Address: 161.53.9.221

The article was downloaded on 22/10/2010 at 16:56

Please note that terms and conditions apply. 


\title{
Semiclassical description of collisionally induced rainbow satellites: a model study
}

\author{
Robert Beuc, Berislav Horvatić and Mladen Movre \\ Institute of Physics, Bijenička cesta 46, P O Box 304, HR-10001 Zagreb, Croatia
}

Received 13 July 2010, in final form 14 July 2010

Published 22 October 2010

Online at stacks.iop.org/JPhysB/43/215210

\begin{abstract}
We apply the uniform Airy approximation (Beuc and Horvatic 1992 J. Phys. B: At. Mol. Opt. Phys. 25 1497) to the analytical model proposed by Devdariani et al (2002 J. Phys. B: At. Mol. Opt. Phys. 35 2469). The model describes asymptotically forbidden quasimolecular optical transitions within the frame of the semiclassical approach. Our results support conclusions that the uniform Airy approximation, originally developed for the asymptotically allowed transitions, is applicable to a broad class of asymptotically forbidden but collisionally assisted optical transitions as well. In addition, we present a new transitional formula and discuss some further approximations.
\end{abstract}

\section{Introduction}

A process of the type

$$
\mathrm{A}+\mathrm{B}+\hbar \omega \leftrightarrow \mathrm{A}^{*}+\mathrm{B},
$$

where during the collision a pair of atoms $\mathrm{A}+\mathrm{B}\left(\right.$ or $\left.\mathrm{A}^{*}+\mathrm{B}\right)$, forming a quasimolecule, absorbs (or emits) a photon and undergoes a transition into another electronic state, with one of the atoms (the 'perturber') finally emerging in its initial state, is usually called an optical collision.

In the adiabatic approximation, it is assumed that collisions do not produce any nonradiative transitions in the $\mathrm{A}+\mathrm{B}$ (or $\left.\mathrm{A}^{*}+\mathrm{B}\right)$ quasimolecule. The main features of the spectrum are well described within the two-level approximation and the rotating-wave approximation [1, 2]. If the relative motion of colliding atoms is described semiclassically, the first-order perturbation result for the transition amplitude (up to a constant phase factor) can be written in the form

$$
\begin{aligned}
& a(\Delta \omega)=\mathrm{i} \int_{-\infty}^{+\infty} \mathrm{d} t \Omega_{\mathrm{R}}[R(t)] \\
& \quad \times \exp \left[\frac{\mathrm{i}}{\hbar} \int_{0}^{t} \mathrm{~d} t^{\prime}\left\{\Delta U\left[R\left(t^{\prime}\right)\right]-\hbar \Delta \omega\right\}\right] .
\end{aligned}
$$

Here, $\Omega_{\mathrm{R}}(R)$ is the quasimolecular Rabi frequency at the internuclear separation $R=R(t)$, defined by

$$
\Omega_{\mathrm{R}}(R)=\frac{1}{2 \hbar} \vec{D}(R) \cdot \overrightarrow{\mathrm{E}}_{0},
$$

where $\overrightarrow{\mathrm{E}}_{0}$ is the amplitude of the electric field $\overrightarrow{\mathrm{E}}=\overrightarrow{\mathrm{E}}_{0} \cos (\omega t)$ and $\vec{D}(R)$ is the matrix element of the quasimolecular transition dipole moment. $\Delta U(R)=\Delta V(R)-\Delta V(\infty)$, where $\Delta V(R)=V^{*}(R)-V_{0}(R)$ is the difference potential, $V^{*}(R)$ and $V_{0}(R)$ being the energy curves for the excited and ground quasimolecular electronic state, respectively. The frequency shift $\Delta \omega=\omega-\omega_{0}$ is defined with respect to the unperturbed frequency $\omega_{0}=\Delta V(\infty) / \hbar$, and $R(t)$ is a classical trajectory for some impact parameter $b$ and single collision energy $E$.

The time integral in equation (2) refers to a complete collision, but it can be analyzed in terms of two half-collisions, each half ending (the incoming one) or starting (the outgoing one) at the minimum internuclear distance $R_{\min }$ attained in the collision, $R_{\min }=R(0)$. The transition amplitude (2) is $a=a_{\mathrm{in}}+$ $a_{\text {out }}$, where $a_{\text {in }}\left(a_{\text {out }}\right)$ is the transition amplitude describing the incoming (outgoing) half-collision. With the assumption that the incoming and outgoing halves are kinematically identical (i.e. $R(-t)=R(t))$, one has $a_{\text {in }}=-a_{\text {out }}^{*}$. For convenience, the time variable can be shifted (for each half separately) so that the extremal point $R_{0}$ of $\Delta U(R)$ is reached for $t=0$, i.e. $R(0)=$ $R_{0}$. The change of variable from $t$ to $R(t)$ then yields

$$
\begin{gathered}
a_{\text {out }}(\Delta \omega)=\mathrm{i} \int_{R_{\min }}^{\infty} \mathrm{d} R G(R) \mathrm{e}^{\mathrm{i} F\left(R, R_{\min }, \Delta \omega\right)}, \\
F\left(R, R_{\min }, \Delta \omega\right)=\frac{1}{\hbar} \int_{R_{\min }}^{R} \frac{\mathrm{d} R^{\prime}}{v_{\mathrm{r}}\left(R^{\prime}\right)}\left[\Delta U\left(R^{\prime}\right)-\hbar \Delta \omega\right], \\
G(R)=\frac{\Omega_{\mathrm{R}}(R)}{v_{\mathrm{r}}(R)},
\end{gathered}
$$

where $v_{\mathrm{r}}[R(t)]=\mathrm{d} R(t) / \mathrm{d} t=\dot{R}[R(t)]$ is the radial relative velocity expressed as a function of $R(t)$. 
The absorption coefficient of the mixture of the atoms of kind $\mathrm{A}$ and atoms of kind $\mathrm{B}$ in a gas cell at temperature $T$ is proportional to the averaged spectrum $\left\langle|a(\Delta \omega)|^{2}\right\rangle_{b, E}$, where the average is over impact parameters $b$ and collision energies $E$, assuming a Maxwellian distribution over energies. (The averaging over the dipole moment orientation is also assumed.)

The pressure broadening and shift of atomic spectral lines can be analyzed in terms of optical collisions. The interaction between the absorbing (or emitting) atom $\mathrm{A}$ and perturbers B shifts the levels of A and produces absorption (or emission) at frequencies that a free atom cannot absorb (or emit). Even in the case of asymptotically forbidden transitions the collisionally assisted optical transitions will occur.

The optical transitions occur in good approximation at Condon points $R_{\mathrm{c}}$ defined as the roots of the equation $\Delta U(R)=$ $\hbar \Delta \omega$. If the radial kinetic energy $\varepsilon_{\mathrm{r}}(R)$ can be assumed to be constant in the vicinity of a point $R_{0}$ which is the most significant for the spectrum formation, the averaging over the impact parameters can be performed analytically and the averaged spectrum takes the form [3-5]

$$
\left\langle|a(\Delta \omega)|^{2}\right\rangle_{b, E} \propto \int_{0}^{\infty} \mathrm{d} x \mathrm{e}^{-x}\left|a\left(\Delta \omega ; \varepsilon_{\mathrm{r}}=k T x, b=0\right)\right|^{2},
$$

i.e. one needs to know only the amplitude for the zero impact parameter.

Devdariani et al [5] obtained a formula that describes asymptotically forbidden quasimolecular optical transitions in the frame of the semiclassical approach. They pointed out the difficulties in the analytical description of asymptotically forbidden transitions, for example due to the fact that the transition moments in this situation cannot be approximated by constant values as in the case of allowed transitions. For the cases where the potential energy curves and the optical transition probability (or radiation width) can both be reasonably approximated by exponential functions, they derived a closed formula which covers the central part of the spectral line, the vicinity of the extremum and the far wings, and also takes into account the fast exponential change in the state radiative width. The formula was obtained with the use of the Morse potential for the potential energy difference and a simple exponential function for the radiation width, assuming a head-on collision (i.e. the zero impact parameter) and a straight-line trajectory.

Analytic models have their indisputable merits, the most prominent one being that they might have exact solutions, which is always most welcome. The drawback is their limited applicability - to only a number of cases for which they constitute a reasonable approximation. On the other hand, general methods applicable to a wider class of cases have obvious advantages, but being approximate by their very nature, they also have limitations of their own.

In this work, a detailed analysis was performed of the applicability of various asymptotic approaches to the analytically solvable Devdariani model [5]. The analyticity of the model enables both obtaining the approximate asymptotic results in the closed (analytic) form and checking them against the exact solution. The methods were tested, appraised and 'practised' on this particular model with the aim of their further application to a wider class of analogous problems, in particular to the profiles of line wings and rainbow satellites originating from the extrema of difference-potential curves.

Our approach to the model case [5] is based on the application of the uniform Airy approximation $[6,7]$ to the integral in equation (4). This approximation was originally applied to the asymptotically allowed transitions [8-10]. The essential point of our approach is a careful treatment of the phases corresponding to the relevant Condon points and the resulting interference effects, as emphasized by Beuc and Horvatić [10], which at the same time takes account of the variable transition moment in the vicinity of an extremum of the curve $\Delta U(R)$ describing the difference between the electronic energies of the initial and final states of the optical transition. The distinction among previous approaches is that Beuc and Horvatić [10] did not invoke local approximations to the phase differences in order to perform the analytical averaging of the spectrum.

\section{Model}

Any $\Delta U(R)$ with an extremum in $R=R_{0}$ can be written in the form

$$
\Delta U(R)=\Delta U\left(R_{0}\right) \Delta \tilde{U}\left[\alpha_{R}\left(R-R_{0}\right)\right],
$$

where $1 / \alpha_{R}$ is a characteristic length, measuring the 'width' of the extremum. We also write

$$
\Omega_{\mathrm{R}}(R)=\gamma_{R} \tilde{\Omega}\left[\beta_{R}\left(R-R_{0}\right)\right],
$$

where $1 / \beta_{R}$ is the characteristic length scaling the variation of $\Omega_{\mathrm{R}}(R)$ (at least in the relevant vicinity of the extremum of $\Delta U(R))$.

Using the dimensionless variable $x=\alpha_{R}\left(R-R_{0}\right)$, we obtain

$$
\begin{gathered}
a_{\text {out }}(\Delta \omega)=\mathrm{i} \frac{\gamma_{R}}{\alpha_{R}} \int_{x_{\min }}^{\infty} \mathrm{d} x \tilde{G}(x, \rho) \mathrm{e}^{\mathrm{i} \tilde{F}\left(x, x_{\min }, \varpi\right)}, \\
\tilde{F}\left(x, x_{\min }, \varpi\right)=\frac{\Delta U\left(R_{0}\right)}{\hbar \alpha_{R}} \int_{x_{\min }}^{x} \frac{\mathrm{d} x^{\prime}}{\tilde{v}_{\mathrm{r}}\left(x^{\prime}\right)}\left[\Delta \tilde{U}\left(x^{\prime}\right)-\varpi\right], \\
\tilde{G}(x, \rho)=\frac{g(x, \rho)}{\tilde{v}_{\mathrm{r}}(x)},
\end{gathered}
$$

where $g(x, \rho)=\tilde{\Omega}(\rho x), x_{\min }=\alpha_{R}\left(R_{\min }-R_{0}\right)$ and $\tilde{v}_{\mathrm{r}}(x) \equiv$ $v_{\mathrm{r}}\left[R_{0}+\left(x / \alpha_{R}\right)\right]=v_{\mathrm{r}}(R)$.

For a constant radial relative velocity, $v_{\mathrm{r}}(R)=v$, the transition amplitude simplifies to

$a_{\text {out }}(\Delta \omega)=\mathrm{i} \frac{\hbar \gamma_{R}}{\Delta U\left(R_{0}\right)} \mathrm{e}^{-\mathrm{i} \lambda f\left(x_{\min }, \varpi\right)} \lambda \int_{x_{\min }}^{\infty} \mathrm{d} x g(x, \rho) \mathrm{e}^{\mathrm{i} \lambda f(x, \varpi)}$,

where $f(x, \varpi)$ is the 'phase function', defined as the primitive function of $\Delta \tilde{U}(x)-\varpi$,

$$
\int_{x_{0}}^{x} \mathrm{~d} x^{\prime}\left[\Delta \tilde{U}\left(x^{\prime}\right)-\varpi\right]=f(x, \varpi)-f\left(x_{0}, \varpi\right),
$$

or, equivalently, $\partial f(x, \varpi) / \partial x \equiv f^{(1)}(x, \varpi)=\Delta \tilde{U}(x)-\varpi$. Clearly, $f^{(1)}(x, \varpi)=0$ if and only if $\Delta \tilde{U}(x)-\varpi=0$, which 
means that the Condon points are determined as the zeros of $f^{(1)}(x, \varpi)$.

The dimensionless parameters $\rho, \lambda$ and $\varpi$ are defined as

$$
\begin{gathered}
\rho=\frac{\beta_{R}}{\alpha_{R}}, \\
\lambda=\frac{\Delta U\left(R_{0}\right)}{\hbar \alpha_{R} v}, \\
\varpi=\frac{\hbar \Delta \omega}{\Delta U\left(R_{0}\right)} .
\end{gathered}
$$

If the difference potential and the Rabi frequency are both due to the exchange interaction, then $\alpha_{R}$ and $\beta_{R}$ are usually of the same order of magnitude, which makes $\rho \sim 1$. The parameter $\lambda$ is specific in that, in addition to the shape parameters $\Delta U\left(R_{0}\right)$ and $\alpha_{R}$ of the difference potential, it depends on the collision velocity $v$ as well, as $\lambda \propto 1 / v$.

As regards the assumption $v_{\mathrm{r}}(R)=$ const., the most usual (and commonly referred to) circumstance for this is the case of a straight-line trajectory (at least locally, where it matters) with the zero impact parameter, $b=0$, but it can fortuitously be satisfied for other special circumstances as well.

In equation (10), we further take the limit $x_{\min } \rightarrow-\infty$, which amounts to a purely formal extension of the integration over $R$ to values $0 \leqslant R<R_{\min }$, inaccessible in a collision, as well as to $-\infty<R<0$, that are simply unphysical. This could make sense if $-x_{\min } \gg 1$, which is fulfilled if the configuration of the relevant potentials happens to be such that $R_{\text {min }}$ is 'far enough' (to the left) from the relevant vicinity of the extremum of $\Delta U(R)$, centred around $R_{0}$ and of the width $\sim 1 / \alpha_{R}$, i.e. if $\left(R_{0}-R_{\min }\right) \gg 1 / \alpha_{R}$. Even so, it is another matter whether the artificially added (unphysical) contribution $\int_{-\infty}^{x_{\min }} \mathrm{d} x$ is also negligible with respect to the physical one, $\int_{x_{\min }}^{+\infty} \mathrm{d} x$. This depends on the actual behaviour of the particular integrand for $-\infty<x \ll-1$, and should be checked for any particular functions $g(x, \rho)$ and $f(x, \varpi)$.

The model difference potential used in [5] is given by the Morse potential

$$
\Delta \tilde{U}(x)=2 \mathrm{e}^{-x}-\mathrm{e}^{-2 x},
$$

and the Rabi frequency $\Omega_{\mathrm{R}}(R)$ is also given by an exponential approximation:

$$
g(x, \rho)=\tilde{\Omega}(\rho x)=\mathrm{e}^{-\rho x} .
$$

Inserting $\Delta \tilde{U}(x)$ given by (13) into the integral (11), we obtain the model phase function

$$
f(x, \omega)=-2 \mathrm{e}^{-x}+\frac{1}{2} \mathrm{e}^{-2 x}-\omega x .
$$

Provided that $-x_{\min }=\alpha_{R}\left(R_{0}-R_{\min }\right) \gg 1$, then for the model functions (13) and (14) one can take the limit $x_{\min } \rightarrow-\infty$, with the resulting integral converging at both ends, if $0<\rho<2$. This 'theoretically allowed' range of $\rho$ is not bad at all since the physically realistic values of $\rho$ are likely to fall somewhere around unity (e.g. if the difference potential and the Rabi frequency are both due to the exchange interaction).

With further applications in mind we note that, for the particular choice of the Morse function (13) to model the difference potential, any function $g(x, \rho)$ increasing more slowly than $\mathrm{e}^{-2 x}=\mathrm{e}^{2|x|}$ for $x \rightarrow-\infty$ and decreasing to 0 faster than $x^{-\rho}, \rho>0$, for $x \rightarrow+\infty$, would do just as well.

With the model functions (13) and (14), $x_{\min } \rightarrow-\infty$, and for $0<\rho<2$ the integral in equation (10) can be calculated in the closed form [5], and the final formula reads

$$
\begin{aligned}
& a_{\text {out }}(\Delta \omega)=\mathrm{i} \frac{\hbar \lambda \gamma_{R}}{\Delta U\left(R_{0}\right)} \lambda^{-\frac{\mathrm{i} \Omega}{2}} \Gamma(\mathrm{i} \Omega) \\
& \quad \times D_{-\mathrm{i} \Omega}\left(2 \lambda^{\frac{1}{2}} \mathrm{e}^{\frac{3 \pi}{4} \mathrm{i}}\right) \mathrm{e}^{-\mathrm{i} \lambda f_{\min }} \mathrm{e}^{-\mathrm{i} \lambda} \mathrm{e}^{-\frac{\pi}{4} \Omega},
\end{aligned}
$$

where $\Omega=\lambda \varpi-\mathrm{i} \rho$ and $\Gamma(z)$ and $D_{-\mathrm{i} \Omega}(z)$ are the gamma and the parabolic cylinder functions, respectively [11]. This exact analytic solution enables the check of the approximate results obtained by any general but approximate methods.

\section{Asymptotic expansion of integrals}

The integral in equation (10) is already written in the form of a Fourier integral,

$$
I(\varpi ; \lambda, \rho)=\int_{-\infty}^{\infty} \mathrm{d} x g(x, \rho) \mathrm{e}^{\mathrm{i} \lambda f(x, \varpi)},
$$

well suited for the asymptotic analysis [12]. The parameter $\lambda$ plays the role of the asymptotic parameter. Both $g(x, \rho)$ and $f(x, \varpi)$ assume real values on the real axis and $\varpi$ is a real parameter. If $\lambda$ is large enough, say $\lambda \geqslant \lambda_{\rho}$, the rapid oscillations of $\exp [i \lambda f(x, \varpi)]$ tend to cancel the contributions to the integral, so the dominant contributions to the asymptotic expansion of $I$ are determined by the behaviour of $f$ in the small neighbourhood of the stationary phase points $x_{i} \equiv x_{i}(\varpi)$, i.e. the roots of $f^{(1)}(x, \varpi)=0$.

For the model phase function $f(x, \varpi)$ given by equation (15), two sets of (complex) solutions of $f^{(1)}(x, \varpi)=$ 0 emerge,

$$
\begin{aligned}
x_{ \pm}^{(k)}(\varpi) & =\operatorname{Ln}\left(\frac{1 \pm \sqrt{1-\varpi}}{\varpi}\right) \\
& =\ln \left(\frac{1 \pm \sqrt{1-\varpi}}{\varpi}\right)+\mathrm{i} 2 k \pi, \quad k=0, \pm 1, \pm 2, \ldots,
\end{aligned}
$$

where the labels \pm are chosen such that $\operatorname{Re} x_{-}^{(k)}(\varpi)<$ $\operatorname{Re} x_{+}^{(k)}(\varpi)$ for any complex $\varpi$ except for the real values $\varpi \geqslant 1$, for which $\operatorname{Re} x_{-}^{(k)}(\varpi)=\operatorname{Re} x_{+}^{(k)}(\varpi)$. The two families of solutions $x_{-}^{(k)}(\varpi)$ and $x_{+}^{(k)}(\varpi)$ are also referred to as the 'inner or left branch' and the 'outer or right branch', respectively, alluding to their 'stemming from' the corresponding parts of the difference potential for $R<R_{0}$ and $R>R_{0}$. This attribution, however, makes sense only where $x_{ \pm}^{(k)}(\varpi)$ are real, i.e., only for real $\varpi$ and for $x_{-}^{(0)}(\varpi \leqslant 1)$ and $x_{+}^{(0)}(0<\varpi \leqslant 1)$. More generally, and for a complex $\varpi$ as well, the \pm branches stem from those of the double-valued function $z^{1 / 2}= \pm \sqrt{z}$, in addition to those stemming from $\operatorname{Ln}(z)$, labelled by the superscript $(k)$, with which they should not be confused.

Of the solutions on or nearest to the real axis, those two that are found to give the relevant contributions to the integral (17) belong to the principal $(k=0)$ branch of $\mathrm{Ln}$ in 


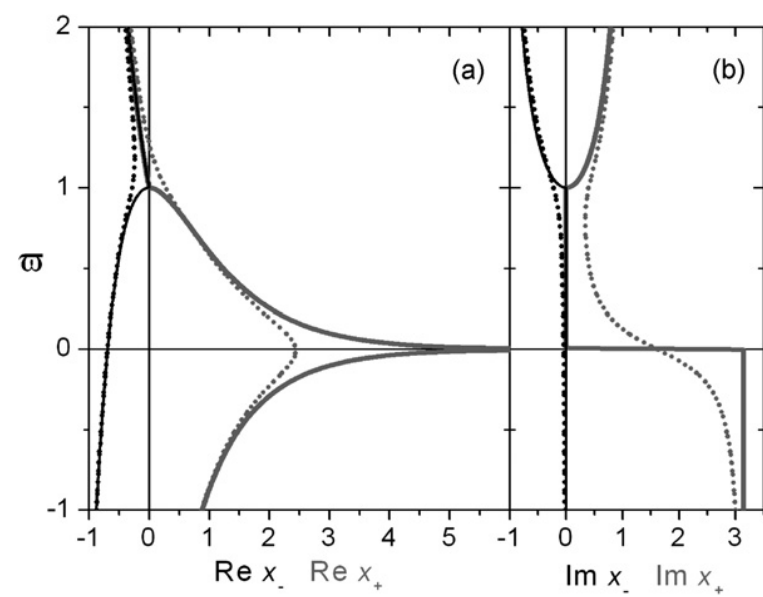

Figure 1. Trajectories of the two stationary points $x_{ \pm}\left(\varpi ; \varpi_{\mathrm{I}}\right)$ as functions of $\varpi$ for two different values of $\varpi_{\mathrm{I}}$. (a) Real part of $x_{+}$, (b) imaginary part of $x_{ \pm}$. Black curves: $x_{-}$('the inner or left branch'), grey curves: $x_{+}$('the outer or right branch'). Solid lines: RFSPs $\left(\varpi_{\mathrm{I}}=0\right)$, dotted lines: CFSPs for $\varpi_{\mathrm{I}}=0.175$. In (a) the RFSPs $x_{-}(\varpi \leqslant 1)$ and $x_{+}(0<\varpi \leqslant 1)$ delineate $\Delta \tilde{U}(x)$.

equation (18), with $-\pi<\operatorname{Im} x_{ \pm}^{(0)}(\varpi) \leqslant \pi$. In further text, we denote them simply by $x_{ \pm}(\varpi)$.

If $\varpi$ is real, then for $\varpi<0$ there is a single (first-order) real stationary point, $x_{-}(\varpi)$, for $0<\varpi<1$ there are two (firstorder) real stationary points, $x_{ \pm}(\varpi)$, merging into a single (second-order) one $x_{0}=0$ for $\varpi \rightarrow 1$, and for $\varpi>1$ there are no real stationary points at all, but a complex conjugate pair of (first-order) stationary points $x_{ \pm}(\varpi)$, merging into $x_{0}$ for $\varpi \rightarrow 1$.

The integrand in (17) can also be rearranged into a slightly different form:

$$
I(\varpi ; \lambda, \rho)=\int_{-\infty}^{\infty} \mathrm{d} x \mathrm{e}^{\mathrm{i} \lambda f(x, \varpi-\mathrm{i} \rho / \lambda)},
$$

amounting to simultaneous substitutions $g(x, \rho) \rightarrow 1$ and $\varpi \rightarrow \varpi-\mathrm{i} \varpi_{\mathrm{I}}$, with $\varpi_{\mathrm{I}}=\rho / \lambda$. The stationary points of the new phase function in $\left(17^{\prime}\right), f\left(x, \varpi-\mathrm{i} \varpi_{\mathrm{I}}\right)$, i.e. the roots of $f^{(1)}\left(x, \varpi-\mathrm{i} \varpi_{\mathrm{I}}\right)=0$, are still given by equation (18), but with a complex parameter $\varpi-i \varpi_{\text {I }}$ instead of a real $\varpi$. As a consequence, they are now all complex for any $\varpi$, as long as $\varpi_{\mathrm{I}} \neq 0$. Figure 1 shows the trajectories of the two stationary points $x_{ \pm}\left(\varpi ; \varpi_{\mathrm{I}}\right)$ on or nearest to the real axis as the functions of the real variable $\varpi$ for two different values of $\varpi_{\mathrm{I}}, 0$ and 0.175 . These two $x_{ \pm}\left(\varpi ; \varpi_{\mathrm{I}}\right)$ are the ones used in the asymptotic treatment of the integrals (17) or $\left(17^{\prime}\right)$. (We put $\varpi$ on the vertical axis so that the stationary points, when real, delineate the shape of the difference potential (13).) In the case $\varpi_{\mathrm{I}}=0$ both points are real for $0 \leqslant \varpi \leqslant 1$ and they coalesce for $\varpi=1$. For $\varpi>1$, they are the complex conjugate pair. For $\varpi \rightarrow 0^{+}$the point $x_{+}(\varpi)$ wanders off to infinity, but for $\varpi<0$ the point $x_{-}(\varpi)$ remains real while $x_{+}(\varpi)$ becomes complex, acquiring a constant imaginary part equal to $\pi$. For $\varpi_{\mathrm{I}} \neq 0$ both stationary points are complex for any $\varpi$, and for $\varpi=1$ they do not coalesce. For further convenience, we introduce abbreviations RFSP (real-frequency stationary point) and CFSP (complex-frequency stationary point) for the cases $\varpi_{\mathrm{I}}=0$ and $\varpi_{\mathrm{I}} \neq 0$, respectively, where 'frequency' refers to the dimensionless complex parameter $\varpi-\mathrm{i} \varpi_{\mathrm{I}}$. Also, we will generally suppress the $\varpi$ dependence of $x_{ \pm}(\varpi)$.

As long as the Condon points are far from the turning point $R_{\min }$ (i.e. for $-x_{\min } \gg 1$ ), the contributions to the transition probability from both half-collisions may be added incoherently, i.e. $|a(\Delta \omega)|^{2}=2\left|a_{\text {out }}(\Delta \omega)\right|^{2}$. The interference term does exist as well, but comprises a rapidly oscillating factor $\exp \left[\mathrm{i} 2 \lambda f\left(x_{\min }, \varpi\right)\right]$ (see equation (10)), which averages out to zero. The transition probability $|a(\Delta \omega)|^{2}$ is then proportional to $|I(\varpi)|^{2}$ and we find it convenient to define the reduced quantity

$$
P(\varpi)=\frac{\lambda}{2 \pi}|I(\varpi)|^{2},
$$

referred to as 'profile' for short in the remaining text. (Here and in the following, we suppressed the explicit dependence on $\lambda$ and $\rho$.)

\subsection{Stationary phase approximation}

If the stationary points $x_{i}$ are 'well enough' isolated from each other, the leading asymptotic contribution to the integral (17) is of the form

$$
I(\varpi)=\sum_{i} I_{i}(\varpi),
$$

where for a first-order ('simple') stationary point $x_{i}$,

$$
I_{i}(\varpi) \equiv I_{i}^{q}(\varpi)=\sqrt{\frac{2 \pi \mathrm{i}}{\lambda}} g\left(x_{i}\right) \frac{\exp \left[\mathrm{i} \lambda f\left(x_{i}\right)\right]}{\sqrt{f^{(2)}\left(x_{i}\right)}},
$$

with the superscript $q$ indicating a quadratic expansion of $f(x)$ about the stationary point $x_{i}$.

If there are no real stationary points, the integral (17) is exponentially small. If a stationary point is complex, its contribution is again given by (21), but only those $x_{i}$ with Im $f\left(x_{i}\right)>0$ (i.e. those that give exponentially decreasing contributions to (17)) can be of use (are 'admissible') for obtaining the asymptotic expansion [12]. The fact that an asymptotic series of a function has a different form in different sectors of the complex plane is known as the Stokes phenomenon $[12,13]$.

In our model case the leading contributions (21) to the amplitude (20) can be evaluated in the closed form, and whenever both stationary points contribute to the spectrum (and are well separated), the amplitude is explicitly given as

$$
I^{q}(\varpi)=I_{+}^{q}(\varpi)+I_{-}^{q}(\varpi),
$$

where

$$
\begin{aligned}
& I_{ \pm}^{q}(\varpi)=\sqrt{\frac{\mp \mathrm{i} \pi}{\lambda \sqrt{1-\varpi}}}\left(\frac{1 \pm \sqrt{1-\varpi}}{\varpi}\right)^{\frac{1}{2}-\mathrm{i} \lambda \varpi-\rho} \\
& \quad \times \exp \left[-\mathrm{i} \lambda\left(\frac{\varpi}{2}\right) \frac{3 \pm \sqrt{1-\varpi}}{1 \pm \sqrt{1-\varpi}}\right]
\end{aligned}
$$

or

$$
\begin{aligned}
& I_{ \pm}^{q}(\varpi)=\sqrt{\frac{\mp \mathrm{i} \pi}{\lambda \sqrt{1-\varpi+\mathrm{i} \rho / \lambda}}}\left(\frac{1 \pm \sqrt{1-\varpi+\mathrm{i} \rho / \lambda}}{\varpi-\mathrm{i} \rho / \lambda}\right)^{\frac{1}{2}-\mathrm{i} \lambda \varpi-\rho} \\
& \quad \times \exp \left[-\mathrm{i} \lambda\left(\frac{\varpi-\mathrm{i} \rho / \lambda}{2}\right) \frac{3 \pm \sqrt{1-\varpi+\mathrm{i} \rho / \lambda}}{1 \pm \sqrt{1-\varpi+\mathrm{i} \rho / \lambda}}\right]
\end{aligned}
$$


for RFSPs and CFSPs, respectively. Note that although the integrals (17) and $\left(17^{\prime}\right)$ are equal, their leading asymptotic terms (23) and $\left(23^{\prime}\right)$ are not.

The simple stationary phase method (for two wellseparated Condon points) yields

$$
P^{q}(\varpi)=\frac{\lambda}{2 \pi}\left|I^{q}(\varpi)\right|^{2}=P_{-}^{q}(\varpi)+P_{+}^{q}(\varpi)+P_{\text {int }}^{q}(\varpi),
$$

where

$$
P_{ \pm}^{q}(\varpi)=\frac{\lambda}{2 \pi}\left|I_{ \pm}^{q}(\varpi)\right|^{2}=\frac{\left|g\left(x_{ \pm}\right)\right|^{2}}{\left|f^{(2)}\left(x_{ \pm}\right)\right|} \mathrm{e}^{-2 \lambda \operatorname{Im} f\left(x_{ \pm}\right)}
$$

and

$$
\begin{aligned}
& P_{\text {int }}^{q}(\varpi)=\frac{\lambda}{\pi} \operatorname{Re}\left\{I_{-}^{q}(\varpi)\left[I_{+}^{q}(\varpi)\right]^{*}\right\} \\
& =2 \sqrt{P_{-}^{q}(\varpi) P_{+}^{q}(\varpi)} \cos \left\{\lambda \operatorname{Re}\left[f\left(x_{+}\right)-f\left(x_{-}\right)\right]\right. \\
& \left.\quad-\frac{1}{2}\left(\varphi_{+}-\varphi_{-}\right)+\left(\vartheta_{+}-\vartheta_{-}\right)\right\},
\end{aligned}
$$

with $\varphi_{ \pm}$and $\vartheta_{ \pm}$denoting the phases of the complex quantities $f^{(2)}\left(x_{ \pm}\right)$and $g\left(x_{ \pm}\right)$, respectively. For two real stationary points $(0<\varpi<1)$, equation (26) simplifies to

$$
\begin{aligned}
& P_{\text {int }}^{q}(\varpi)=2 \operatorname{sgn}\left[g\left(x_{-}\right) g\left(x_{+}\right)\right] \\
& \quad \times \sqrt{P_{-}^{q}(\varpi) P_{+}^{q}(\varpi)} \sin \left\{\lambda\left[f\left(x_{+}\right)-f\left(x_{-}\right)\right]\right\} .
\end{aligned}
$$

The first two terms on the right-hand side of equation (24) represent the incoherent contribution of the two stationary points, $P_{-}^{q}(\varpi)+P_{+}^{q}(\varpi)$, and (after averaging) yield the usual quasistatic approximation of the spectrum [2], whereas the third one, $P_{\text {int }}^{q}(\varpi)$, represents the interference contribution, which in the random-phase approximation averages out to zero. The expression (24) may be called the coherent quasistatic (CQ) approximation. (Note that, at present, we are still dealing with the transition probability for a single collision energy and a single impact parameter.) In order to construct the profile (24), one needs to know only the local values of $f, f^{(2)}$ and $g$ at $x_{ \pm}$. The oscillatory structure is essentially determined by the phase difference $f\left(x_{+}\right)-f\left(x_{-}\right)$.

In the limit $\varpi \rightarrow 0^{+}$, for the RFSP case, $x_{+} \rightarrow+\infty$ and $P_{+}^{q}$ tends to $+\infty$ or 0 for $\rho<1 / 2$ or $\rho>1 / 2$, respectively, as readily seen from equation (23). However, the stationary phase method is not applicable to the region of the 'line core', $|\varpi|<\varpi_{\mathrm{W}}$, where the characteristic parameter $\varpi_{\mathrm{W}}$, known as the Weisskopf limit, is given by $\varpi_{\mathrm{W}}(\lambda)=4[1-\sqrt{1-(1 / 4 \lambda)}] \sqrt{1-(1 / 4 \lambda)}$ for our analytic $\Delta \tilde{U}(x)$. For $\lambda>1 / 2$, one has $\varpi_{\mathrm{W}}(\lambda) \approx 1 / 2 \lambda$ as an excellent approximation.

On the other side of the spectrum, the simple stationary phase result, equations (21) and (22), is divergent for $x_{ \pm} \rightarrow x_{0}$, i.e. for $\varpi \rightarrow \Delta \tilde{U}\left(x_{0}\right) \equiv \varpi_{0}$. (In our model, $x_{0}=0$ and $\varpi_{0}=1$.) The critical value $\varpi_{0}$ may be called the rainbow satellite frequency.

In figure 2, we compare the exact profiles (black solid line) with those obtained by the coherent quasistatic approximation for the RFSPs (grey solid line) and CFSPs (dotted line), for the parameter values $\lambda=1,2,4$ and 8 and $\rho=0.7$ and 0.3 . The shaded strips indicate the corresponding regions of the line

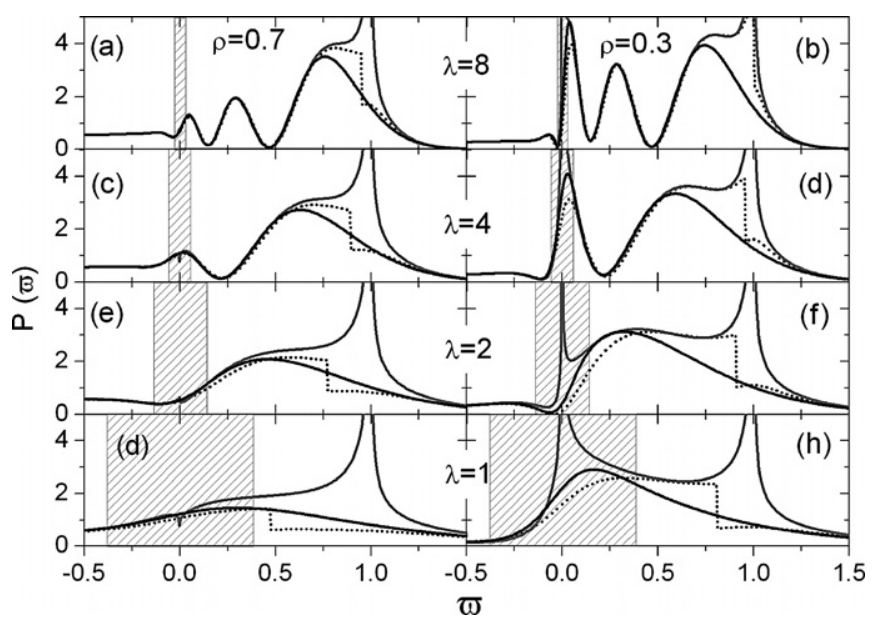

Figure 2. The comparison of the exact profiles (black solid line) with those obtained by the coherent quasistatic approximation for the RFSPs (grey solid line) and CFSPs (dotted line), for the parameter values $\lambda=1,2,4,8$ and $\rho=0.7,0.3$. The shaded strips indicate the corresponding regions of the line core, $|\varpi|<\varpi_{\mathrm{W}}$.

core, $|\varpi|<\varpi_{\mathrm{W}}$, where the stationary phase method is not applicable. Quasistatic profiles deviate from the exact one in the neighbourhood of the line centre bounded by the Weisskopf limits, $|\varpi|<\varpi_{\mathrm{W}}$, where the statistical theory of pressure line broadening is generally inapplicable in the first place, which renders the stationary point method unsuitable as well. In the neighbourhood of the extremum of the differential potential $(\varpi \approx 1)$, the quasistatic approximation gives poor results for the CFSPs (the Stokes phenomenon is clearly visible) and diverges for the RFSPs. For $\varpi<-\varpi_{\mathrm{W}}$, the quasistatic results show excellent agreement with the exact values, as well as for the frequencies in the antistatic region $(\varpi>1)$ greater enough than the extremum frequency $\varpi=1$. In the 'classical' quasistatic region $\varpi_{\mathrm{W}}<\varpi<1$ the coherent quasistatic approximation agrees well with the exact profile, and the region of good agreement widens on both sides with increasing values of $\lambda$, as both the core region and the extremum region shrink.

It is the interference term $P_{\text {int }}^{q}(\varpi)$ that is crucial for producing the correct oscillatory structure of the quasistatic profiles shown in figures 2(a)-(h).

Since we are here primarily interested in the theory of spectral line satellites within the framework of the statistical theory of pressure line broadening, further considerations will be restricted to the spectral region $\varpi>\varpi_{\mathrm{W}}$. This also means leaving out the analysis of some (mathematical) peculiarities arising for $\varpi \leqslant 0$, which, however, are highly interesting in their own right.

\subsection{Uniform Airy approximation}

If for some critical value $\varpi=\varpi_{0}$ two first-order stationary points coalesce into a single second-order one $x_{0}$, then $f^{(1)}\left(x_{0}, \varpi_{0}\right)=f^{(2)}\left(x_{0}\right)=0$ and the leading asymptotic contribution to the integral (17) given by (20) and (21) becomes singular as $\varpi \rightarrow \varpi_{0}$ (due to $f^{(2)}\left(x_{0}\right)=0$ ). 
3.2.1. Uniformly analytic mapping. An efficient way to treat simultaneously the region of $\varpi$ in the neighbourhood of $\varpi_{0}$ as well as the region where two stationary points are well separated is the uniform asymptotic expansion, based on the mapping $[2,6,12]$

$$
\lambda f(x, \varpi)=\frac{1}{3} u^{3}-\zeta(\varpi) u+A(\varpi) .
$$

Here, with

$$
\begin{gathered}
2 A(\varpi)=\lambda\left[f\left(x_{+}\right)+f\left(x_{-}\right)\right], \\
\frac{4}{3}[\zeta(\varpi)]^{3 / 2}=\lambda\left[f\left(x_{+}\right)-f\left(x_{-}\right)\right],
\end{gathered}
$$

the points $x=x_{ \pm}(\varpi)$ correspond to $u=\mp \zeta^{1 / 2}$ and the correspondence $u \leftrightarrow x$ is one to one.

The leading asymptotic contribution to the integral (17) is now [6]

$$
\begin{aligned}
I^{u}(\varpi) & =\sqrt{2} \pi \mathrm{e}^{\mathrm{i} A}\left[\left(\frac{g\left(x_{-}\right)}{\sqrt{\lambda f^{(2)}\left(x_{-}\right)}}+\frac{g\left(x_{+}\right)}{\sqrt{-\lambda f^{(2)}\left(x_{+}\right)}}\right) \zeta^{\frac{1}{4}} \mathrm{Ai}(-\zeta)\right. \\
& \left.-\left(\frac{g\left(x_{-}\right)}{\sqrt{\lambda f^{(2)}\left(x_{-}\right)}}-\frac{g\left(x_{+}\right)}{\sqrt{-\lambda f^{(2)}\left(x_{+}\right)}}\right) \frac{\mathrm{i}}{\zeta^{\frac{1}{4}}} \mathrm{Ai}^{\prime}(-\zeta)\right]
\end{aligned}
$$

where $\operatorname{Ai}(z)$ and $\operatorname{Ai}^{\prime}(z)$ denote the Airy function and its first derivative, respectively, defined as in [11]. We will call equation (30) the uniform Airy (UA) approximation.

In our model case, the uniform mapping parameter $\zeta(\varpi)$ is given by the expression

$$
\zeta(\varpi)=\lambda^{\frac{2}{3}}\left\{\frac{3}{4}\left[2 \sqrt{1-\varpi}-\varpi \ln \left(\frac{1+\sqrt{1-\varpi}}{1-\sqrt{1-\varpi}}\right)\right]\right\}^{\frac{2}{3}}
$$

for the RFSPs. The same expression is valid for the CFSPs as well if on the right-hand side of equation (31) one replaces $\varpi$ with $\varpi-\mathrm{i} \rho / \lambda$. For real $\varpi$ the parameter $\zeta(\varpi)$ is real and negative for $\varpi>1$, real and positive for $0<\varpi \leqslant 1$, but complex-valued for $\varpi<0$. For the phase function $f(x, \varpi-\mathrm{i} \rho / \lambda), \zeta(\varpi)$ is complex-valued for any (real) $\varpi$.

For $|1-\varpi|<1$, the expression (31) can be expanded as

$$
\zeta(\varpi)=\zeta_{t}(\varpi)\left[1+\frac{2}{15}(1-\varpi)+\cdots\right],
$$

where

$$
\zeta_{t}(\varpi)=\lambda^{2 / 3}(1-\varpi)
$$

The expression (33) is obviously a good approximation of the uniform result (31) for $|1-\varpi| \ll 1$ at least, but proves even much better than that.

The profile $P^{u}(\varpi)$ was calculated according to

$$
P^{u}(\varpi)=\frac{\lambda}{2 \pi}\left|I^{u}(\varpi)\right|^{2} .
$$

In figure 3, we compare the exact profiles (black solid line) with those obtained by the UA approximation with RFSPs (grey solid line) and CFSPs (dotted line). The profiles are compared for two values of the transition dipole moment parameter, $\rho=0.7$ (left column) and $\rho=0.3$ (right column), for a series of values of the asymptotic parameter, $\lambda=1,2$, 4,8 . As could be expected, the agreement of the asymptotic results with the exact ones becomes increasingly better as

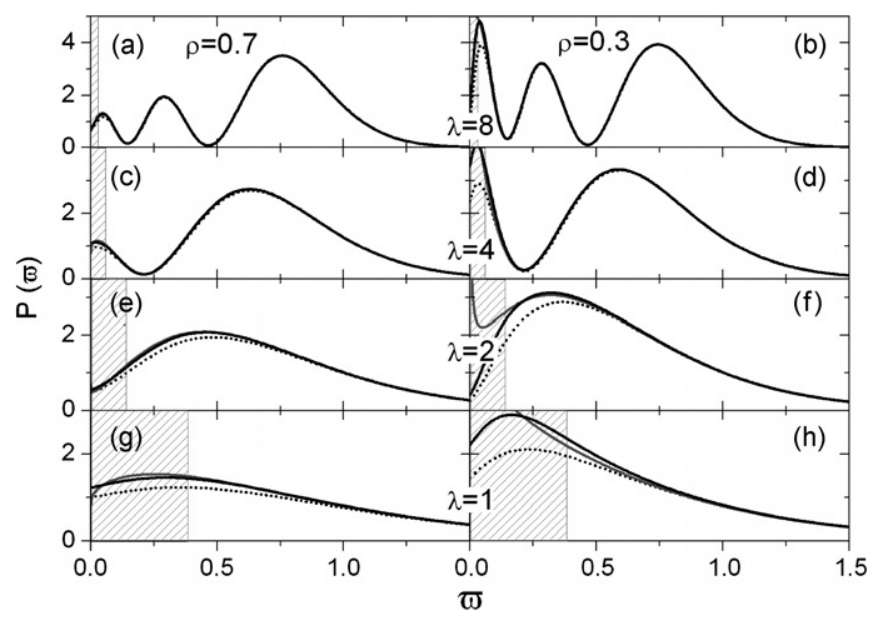

Figure 3. The exact profile (black solid line) compared with those obtained by the UA approximation using uniform mapping of RFSPs (grey solid line) and CFSPs (dotted line). The shaded regions around $\varpi=0$ are defined by the Weisskopf limit. The left column displays profiles for $\rho=0.7$ and the right one for $\rho=0.3$. From top to bottom, the parameter $\lambda$ decreases from 8 to 1 .

$\lambda$ increases. For frequencies $\varpi>\varpi_{W}$, the agreement is complete for $\lambda>2$, but remains satisfactory even for smaller values of $\lambda$.

One can see that the uniform mapping of RFSPs gives better agreement with the exact profile than that of CFSPs in the whole range of its applicability. Also, for the calculation of spectra in a real physical situation it is numerically simpler to work out the real stationary points than the complex ones. In summary, the asymptotic calculation based on the uniform mapping of real-frequency stationary points gives correct results for $\lambda>1$ and also proves more convenient for practical applications.

3.2.2. Transitional Airy approximation. The transitional Airy (TA) approximation $I^{t}(\varpi)$ constitutes the limit of the uniform one valid around the extremum of the difference potential, i.e. for $\varpi \approx \varpi_{0}$, which amounts to $\zeta \rightarrow 0$, $I^{t}\left(\varpi_{0}\right)=I^{u}\left(\varpi_{0}\right)$. The correct form of the transitional Airy approximation is

$$
\begin{aligned}
I^{t}(\varpi) & =2 \pi\left(\frac{2}{\lambda\left|f^{(3)}\left(x_{0}\right)\right|}\right)^{1 / 3} \mathrm{e}^{\mathrm{i} \lambda f\left(x_{0}\right)} g\left(x_{0}\right) \\
\times & {\left[\operatorname{Ai}\left(-\zeta_{t}\right)+\mathrm{i}\left(\frac{2}{\lambda\left|f^{(3)}\left(x_{0}\right)\right|}\right)^{1 / 3}\right.} \\
\times & \left.\left(\frac{g^{(1)}\left(x_{0}\right)}{g\left(x_{0}\right)}-\frac{f^{(4)}\left(x_{0}\right)}{6 f^{(3)}\left(x_{0}\right)}\right) \mathrm{Ai}^{\prime}\left(-\zeta_{t}\right)\right],
\end{aligned}
$$

where

$$
\zeta_{t}=\lambda^{2 / 3}\left(\frac{2}{\left|f^{(3)}\left(x_{0}\right)\right|}\right)^{1 / 3} f^{(1)}\left(x_{0}, \varpi\right)
$$

and $f^{(1)}\left(x_{0}, \varpi\right)=\Delta \tilde{U}\left(x_{0}\right)-\varpi=\varpi_{0}-\varpi$.

The TA approximation $I^{t}(\varpi)$ reproduces $I^{u}(\varpi)$ on the neighbourhood of $\varpi \approx \varpi_{0}$ and enables analytical continuation from the region $\varpi<\varpi_{0}$ into the region $\varpi>\varpi_{0}$. The coefficient preceding $\mathrm{Ai}^{\prime}$ comprises information about the 
rate of change of the transition dipole moment $\left[g^{(1)}\left(x_{0}\right)\right]$ as well as the anharmonicity of the difference potential (contained in $\left.f^{(4)}\left(x_{0}\right)\right)$. The TA approximation reduces to what we shall call the extended cubic (EC) approximation [6] whenever

$$
\left|\frac{f^{(4)}\left(x_{0}\right)}{6 f^{(3)}\left(x_{0}\right)}\right| \ll\left|\frac{g^{(1)}\left(x_{0}\right)}{g\left(x_{0}\right)}\right| \text {. }
$$

The simple cubic (SC) approximation is obtained if one altogether neglects the term with $\mathrm{Ai}^{\prime}$ in equation (35). (The hierarchy of approximations may be put into the symbolic form as $\mathrm{SC} \subseteq \mathrm{EC} \subseteq \mathrm{TA}$.)

In the cubic approximation (expansion of $f(x, \varpi)$ around $x_{0}$ ) not only the phase function $f(x, \varpi)$ is approximated by $f_{0}^{c}(x, \varpi)$, a cubic polynomial in $x-x_{0}$, but the stationary points are approximated as well. They are obtained from the approximate condition $\partial f_{0}^{c}(x, \varpi) / \partial x=0$, as $x_{ \pm}^{(0)}-x_{0}=$ $\pm \sqrt{2\left(\varpi-\varpi_{0}\right) / f^{(3)}\left(x_{0}\right)}$, and are symmetric about $x_{0}$. If one takes into account the next term in the Taylor expansion, i.e. $f^{(4)}\left(x_{0}\right) \neq 0$, the stationary points are slightly shifted, $x_{ \pm}^{(1)}-$ $x_{0}=\left(x_{ \pm}^{(0)}-x_{0}\right)\left\{1-\left[f^{(4)}\left(x_{0}\right) / 6 f^{(3)}\left(x_{0}\right)\right]\left(x_{ \pm}^{(0)}-x_{0}\right)+\cdots\right\}$, but their separation $x_{+}^{(1)}-x_{-}^{(1)}$ remains the same as in the cubic approximation within this order of accuracy, the first nonvanishing relative correction being $\propto\left(x_{ \pm}^{(0)}-x_{0}\right)^{2} \propto\left(\varpi_{0}-\right.$ $\varpi)$ and comprising both $f^{(4)}\left(x_{0}\right)$ and $f^{(5)}\left(x_{0}\right)$. As regards $\zeta(\varpi)$, in the cubic approximation it reduces exactly to $\zeta_{t}(\varpi)$ given by equation (36) and the first nonvanishing relative correction with respect to $\zeta_{t}(\varpi)$, linear in $\varpi_{0}-\varpi$, also comprises both $f^{(4)}\left(x_{0}\right)$ and $f^{(5)}\left(x_{0}\right)$.

In our model case one has $x_{0}=0, \varpi_{0}=1, f(0, \varpi)=$ $-3 / 2, f^{(1)}(0, \varpi)=1-\varpi, f^{(3)}(0)=-2, f^{(4)}(0)=6$, so $\zeta_{t}=\lambda^{2 / 3}(1-\varpi)$ (see equation (33)) and

$$
\begin{aligned}
& I^{t}(\varpi)=\frac{2 \pi}{\lambda^{1 / 3}} \mathrm{e}^{-\mathrm{i} \frac{3}{2} \lambda}\left\{\operatorname{Ai}\left[-\lambda^{2 / 3}(1-\varpi)\right]\right. \\
& \left.+\mathrm{i} \frac{1}{\lambda^{1 / 3}}\left(\frac{1}{2}-\rho\right) \mathrm{Ai}^{\prime}\left[-\lambda^{2 / 3}(1-\varpi)\right]\right\} .
\end{aligned}
$$

For $\varpi=1$, the first term of (38) reproduces the leading contribution of the second-order stationary point $x_{0}(\varpi=1)=$ 0 , as it should. However, since $g^{(1)}(x) / g(x)=-\rho$ (for any $x$ ) and $f^{(4)}(0) /\left[6 f^{(3)}(0)\right]=-1 / 2$, the condition (37) is not fulfilled for our 'allowed range' $0<\rho<2$.

The profile is given by

$$
\begin{aligned}
P^{t}(\varpi) & =2 \pi\left[g\left(x_{0}\right)\right]^{2} \lambda\left(\frac{2}{\lambda\left|f^{(3)}\left(x_{0}\right)\right|}\right)^{2 / 3} \\
\times & \left\{\left[\operatorname{Ai}\left(-\zeta_{t}\right)\right]^{2}+\left(\frac{2}{\lambda\left|f^{(3)}\left(x_{0}\right)\right|}\right)^{2 / 3}\right. \\
\times & {\left.\left[\frac{g^{(1)}\left(x_{0}\right)}{g\left(x_{0}\right)}-\frac{f^{(4)}\left(x_{0}\right)}{6 f^{(3)}\left(x_{0}\right)}\right]^{2}\left[\mathrm{Ai}^{\prime}\left(-\zeta_{t}\right)\right]^{2}\right\}, }
\end{aligned}
$$

which in our model reduces to

$$
P^{t}(\varpi)=2 \pi \lambda^{\frac{1}{3}}\left\{\left[\operatorname{Ai}\left(-\zeta_{t}\right)\right]^{2}+\left(\rho-\frac{1}{2}\right)^{2} \frac{1}{\lambda^{2 / 3}}\left[\operatorname{Ai}^{\prime}\left(-\zeta_{t}\right)\right]^{2}\right\} \text {. }
$$

In the special case $\rho=1 / 2$, the contribution of the $\mathrm{Ai}^{\prime}$ term is absent, and furthermore, the symmetry of $\left(\rho-\frac{1}{2}\right)^{2}$ around $\rho=1 / 2$ has some interesting consequences: the contribution of the $\mathrm{Ai}^{\prime}$ term comes out the same for both the constant dipole moment, $\rho=0$, and a rather strongly variable dipole moment with $\rho=1$, for any $\varpi \approx \varpi_{0}$, and the same holds for our two representative values $\rho=0.7$ and $\rho=0.3$. This contribution is negligible for $\varpi \approx \varpi_{0}$ whenever $\left(\rho-\frac{1}{2}\right)^{2} \ll 1.88 \lambda^{2 / 3}$. We note in passing that Devdariani et al [5] used $\rho=0.54$, the value chosen to describe the specific experimental example they discussed.

\subsubsection{Other approximations. Using the identity}

$$
A=\lambda f\left(x_{ \pm}\right) \mp \frac{2}{3} \zeta^{2 / 3},
$$

following from the defining relations (28) and (29), the expression (30) for $I^{u}$ can be rearranged into the form resembling that of equation (22) [7],

$$
I_{\mathrm{F}}^{u}(\varpi)=I_{-}^{q} J_{\mathrm{F}}^{-}\left(\zeta_{-}\right)+I_{+}^{q} J_{\mathrm{F}}^{+}\left(\zeta_{+}\right),
$$

where $I_{ \pm}^{q}(\varpi)$ are the leading contributions of the first-order stationary points, given by equation (21), the "correction functions' $J_{\mathrm{F}}^{ \pm}(\zeta)$ are defined as

$$
J_{\mathrm{F}}^{ \pm}(\zeta)=\sqrt{\pi} \zeta^{\frac{1}{4}}[\operatorname{Ai}(-\zeta) \mp i \mathrm{~F}(\zeta)] \exp \left[\mp \mathrm{i}\left(\frac{2}{3} \zeta^{\frac{3}{2}}-\frac{\pi}{4}\right)\right],
$$

and where, for future convenience, we added more flexibility by allowing more general arguments $\zeta_{ \pm}$. For the UA approximation one has, of course, $\zeta_{-}=\zeta_{+}=\zeta$, $\mathrm{F}(\zeta)=-\mathrm{Ai}^{\prime}(-\zeta) / \zeta^{1 / 2}$, and $I_{F}^{u}(\varpi)=I^{u}(\varpi)$ as given by equation (30). Alternative methods due to Miller [14] and Bieniek [15] yield $\mathrm{F}(\zeta)=\mathrm{Bi}(-\zeta)$ and $\mathrm{F}(\zeta)=\mathrm{Gi}(-\zeta)$, respectively, where $\operatorname{Bi}(x)$ is the irregular homogeneous Airy function and $\operatorname{Gi}(x)$ the regular inhomogeneous Airy function [11]. Equation (42) with either $\mathrm{F}(\zeta)=\mathrm{Bi}(-\zeta)$ or $\mathrm{F}(\zeta)=$ $\mathrm{Gi}(-\zeta)$ is applicable only for $\zeta \geqslant 0$, whereas the variant with $\mathrm{Ai}^{\prime}$ is valid for $\zeta<0$ as well.

The asymptotic (large $|\zeta|$ ) form of the correction functions $J_{\mathrm{F}}^{ \pm}(\zeta)$ follows from the asymptotic behaviour of $\mathrm{Ai}(-\zeta)$ and $\mathrm{F}(\zeta)$ [11]. In the limit $\zeta \rightarrow \infty$ (two well-separated real stationary points, $f\left(x_{ \pm}\right)$real), the expression (42) goes over smoothly into the quasistatic one (22). For $\zeta \rightarrow-\infty$, the stationary points constitute a complex conjugate pair and $f\left(x_{ \pm}\right)$(and $g\left(x_{ \pm}\right)$as well) are complex-valued. In that case the contribution of the 'admissible' stationary point $\left(\operatorname{Im} f\left(x_{i}\right)>0\right.$, in our case $\left.x_{i}=x_{-}\right)$is damped out exponentially due to $I_{i}^{q} \propto \exp \left(-\lambda\left|\operatorname{Im} f\left(x_{i}\right)\right|\right.$ ) (with $J_{F}^{i}(\zeta$ ) for $\mathrm{F}(\zeta)=-\mathrm{Ai}^{\prime}(-\zeta) / \zeta^{1 / 2}$ remaining around unity) and constitutes the 'antistatic wing' $[2,16]$, while the contribution of the 'inadmissible' one $\left(\operatorname{Im} f\left(x_{j}\right)<0\right.$, in our case $\left.x_{j}=x_{+}\right)$ is suppressed by the quickly decreasing $J_{\mathrm{F}}^{j}(\zeta)$, although $I_{j}^{q} \propto \exp \left(+\lambda\left|\operatorname{Im} f\left(x_{j}\right)\right|\right)$.

In the limit $\zeta \rightarrow 0$, the three variants of equation (42) do not coincide. The choice $\mathrm{F}(\zeta)=-\mathrm{Ai}^{\prime}(-\zeta) / \zeta^{1 / 2}$ (i.e. UA) yields the value obtained from the TA approximation, whereas for both $\mathrm{Bi}$ and $\mathrm{Gi}$ the limit is given by the SC approximation, which in these two cases can be used to analytically continue equation (42) into the region $\varpi>\varpi_{0}$. These continuations are approximate, of course. 
The mapping parameter $\zeta$ can also be approximated locally by the cubic expansion of the phase function around a stationary point [14]. The local approximation implies taking the exact value of the phase at the expansion point but only an approximate phase at the other (also approximately determined) stationary point. More precisely, for the two values of $\zeta=\left\{\frac{3}{4} \lambda\left[f\left(x_{+}\right)-f\left(x_{-}\right)\right]\right\}^{2 / 3}$, appearing as the arguments $\zeta_{\mp}$ of $J_{\mathrm{F}}^{\mp}\left(\zeta_{\mp}\right)$ in expression (42), the phase difference $f\left(x_{+}\right)-f\left(x_{-}\right)$is approximated by $\pm\left[f_{\mp}^{c}\left(x_{ \pm}^{c}\right)-\right.$ $\left.f\left(x_{\mp}\right)\right]$, respectively, where $f_{\mp}^{c}(x)$ are the two cubic expansions of $f(x)$ around the two (exact) stationary points $x_{\mp}$, respectively, and $x_{ \pm}^{c}$ are the approximate 'other' stationary points, obtained from the approximate condition $\partial f_{\mp}^{c}(x) / \partial x=$ 0 . This yields

$$
\zeta_{ \pm}^{l}(\varpi)=\left(\frac{\lambda}{2}\right)^{\frac{2}{3}} \frac{\left[f^{(2)}\left(x_{ \pm}\right)\right]^{2}}{\left[f^{(3)}\left(x_{ \pm}\right)\right]^{\frac{4}{3}}},
$$

and for our model case we have

$$
\zeta_{ \pm}^{l}(\varpi)=\zeta_{t}(\varpi)\left[\frac{1 \mp \sqrt{1-\varpi}}{(1 \mp 2 \sqrt{1-\varpi})^{2}}\right]^{\frac{2}{3}},
$$

with $\zeta_{t}(\varpi)$ given by equation (33). A local parameter $\zeta_{i}^{l}(\varpi)$ will obviously diverge if the respective expansion point $x_{i}$ hits an inflection point of the difference potential since $f^{(3)}\left(x_{\text {infl }}\right)=$ $\Delta \tilde{U}^{(2)}\left(x_{\text {infl }}\right)=0$. This divergence at the inflection point is an intrinsic flaw of the above-described local cubic approximation scheme and can not be remedied (within this very scheme). In our case, $\Delta \tilde{U}(x)$ does have an inflection in its outer branch, in $x=\ln 2$, with $\Delta \tilde{U}(\ln 2)=3 / 4$, so it is $\zeta_{+}^{l}(\varpi)$ that diverges for $\varpi \rightarrow 3 / 4$, as $\zeta_{+}^{l}(\varpi) \rightarrow(4 \lambda)^{2 / 3}\left(\varpi-{ }_{4}^{3}\right)^{-4 / 3}$. Around $\varpi=1$ one finds $\zeta_{ \pm}^{l}(\varpi)=\zeta_{t}(\varpi)[1 \pm 2 \sqrt{(1-\varpi)}+\cdots]$ for $|\sqrt{1-\varpi}| \ll 1$, and since there $|\sqrt{1-\varpi}| \gg|1-\varpi|$, one has $\zeta_{ \pm}^{l}(\varpi) \cong \zeta_{t}(\varpi)$ in a much narrower range of $\varpi$ around $\varpi=1$ than $\zeta(\varpi) \cong \zeta_{t}(\varpi)$.

As for the limit $\zeta_{ \pm}^{l}=0$, the variants of equation (42) using either $\mathrm{Bi}$ or $\mathrm{Gi}$ yield $\mathrm{SC}$, whereas the form with $\mathrm{F}\left(\zeta_{ \pm}^{l}\right)=-\mathrm{Ai}^{\prime}\left(-\zeta_{ \pm}^{l}\right) /\left(\zeta_{ \pm}^{l}\right)^{1 / 2}$ yields EC (not TA!). Here, again, for the choices $\mathrm{Bi}$ or $\mathrm{Gi}$ the form (42) is applicable only for $\varpi<\varpi_{0}$.

Figure 4 displays the real and imaginary parts of the mapping parameter $\zeta(\varpi)$ as functions of (real) $\varpi$ for both choices of the phase function, $f(x, \varpi)$ in $4(a)$ and $f(x, \varpi-$ $\mathrm{i} \rho / \lambda)$ in 4(c). The corresponding parameter of the transitional Airy approximation $\zeta_{t}(\varpi)$ and the local parameters $\zeta_{ \pm}^{l}(\varpi)$, given by equations (33) and (45), respectively, are also shown in 4(b) and 4(d). The three variants of $\zeta(\varpi)$ shown in 4(a), 4(b) and 4(c) exhibit very similar functional behaviour, and $\zeta(\varpi)$ for RFSPs and $\operatorname{Re} \zeta(\varpi)$ for CFSPs are both barely distinguishable from $\zeta_{t}(\varpi)$ in as wide a region as $\frac{1}{2} \leqslant \varpi \leqslant \frac{3}{2}$. Not so with the local parameters $\zeta_{ \pm}^{l}(\varpi)$ shown in 4(d), which are conspicuously different from the previous three forms. They agree with the transitional parameter $\zeta_{t}(\varpi)$ (which also means with $\operatorname{Re} \zeta(\varpi))$ only in an extremely narrow frequency region $|\sqrt{1-\varpi}| \ll 1$ around $\varpi=1$, and everywhere else they are of a profoundly wrong functional form as well as quantitatively very much off target. For $\varpi>1$, they constitute a complex conjugate pair, so their real parts coincide. Note

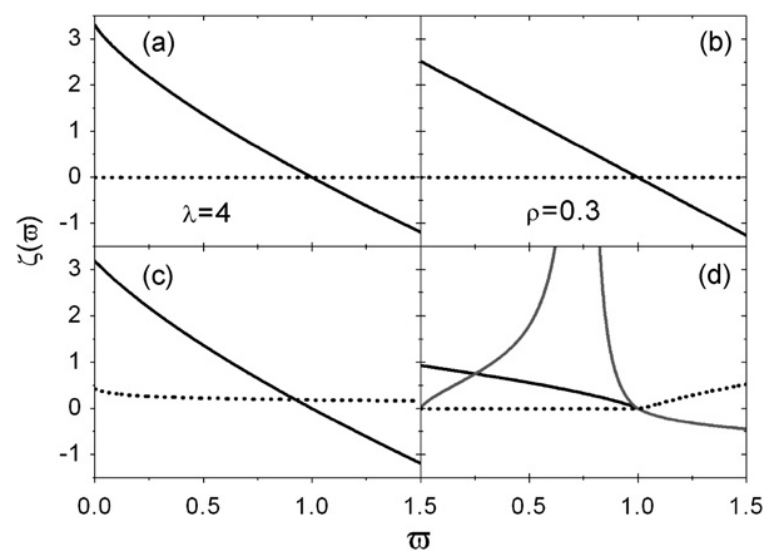

Figure 4. Real (solid line) and imaginary part (dotted line) of the mapping parameter $\zeta(\varpi)$ for special but physically representative values of the parameters $\lambda=4$ and $\rho=0.3$. (a) and (c): uniform Airy approximation for RFSPs and CFSPs, respectively; (b) transitional Airy approximation (for RFSP's); (d) local cubic approximation (for RFSPs)_black solid line: $\operatorname{Re} \zeta_{-}^{l}(\varpi)$, grey solid line: $\zeta_{+}^{l}(\varpi \leqslant 1)$, while for $\varpi>1$ both $\operatorname{Re} \zeta_{ \pm}^{l}(\varpi)$ coincide; dotted line: $\operatorname{Im} \zeta_{+}^{l}(\varpi)=-\operatorname{Im} \zeta_{-}^{l}(\varpi)$.

that although figure 4 shows the mapping parameters for a special (but physically reasonable) value of the parameter $\lambda$, their qualitative relationships remain the same for any value of $\lambda$ since all four expressions for $\zeta(\varpi)$ scale as $\lambda^{2 / 3}$.

As already noted by Miller [14], equation (42) with the local arguments $\zeta_{\mp}=\zeta_{\mp}^{l}$ requires the evaluation of the third derivatives of $f(x)$, in addition to those needed in the simplest approximation (22) or uniform approximations (42) with $\zeta_{\mp}=\zeta=\left\{\frac{3}{4} \lambda\left[f\left(x_{+}\right)-f\left(x_{-}\right)\right]\right\}^{2 / 3}$. However, as the local approximation offers an easy generalization to the cases of more than two Condon points and also has a form suitable for the analytical evaluation of the ensemble average, it was used in various applications (see [2] and references therein).

Figure 5 displays the profiles calculated from equation (42) using either $\zeta_{\mp}=\zeta$ or $\zeta_{\mp}=\zeta_{\mp}^{l}$ as the arguments, for the case of an extremely varying dipole moment (but still in the 'allowed' range), $\rho=1.9$, and $\lambda=8$. The choice $\mathrm{F}\left(\zeta_{ \pm}\right)=\mathrm{Gi}\left(-\zeta_{ \pm}\right)$proves unsatisfactory [17] and is not reported here. In this extreme case, only the UA approximation, $\mathrm{F}\left(\zeta_{\mp}\right)=-\mathrm{Ai}^{\prime}(-\zeta) /(\zeta)^{1 / 2}$, reproduces the exact profile, the two curves being indistinguishable in figure 5(a). The CQ approximation coincides with the uniform approximation based on the Bi function (UB) for $\varpi$ up to $\approx 0.7$, but they both miss the right position of oscillation peaks in the region of interest. However, the greater the parameter $\lambda$, the better the overall agreement. On the other hand, the corresponding uniform approximations using the local arguments $\zeta_{\mp}^{l}$ in equation (42), UAL and UBL, do not fare as well as one would wish.

The function $\zeta_{+}^{l}(\varpi)$ assumes large values in the neighbourhood of the inflection in $\varpi=\frac{3}{4}$, which makes its contribution in that region of a quasistatic form, but an artifactual one. On the other hand, for smaller frequencies $\varpi$ the values of $\zeta_{+}^{l}(\varpi)$ decrease sharply and the profile fails to assume the (factual!) quasistatic form just where it should. 


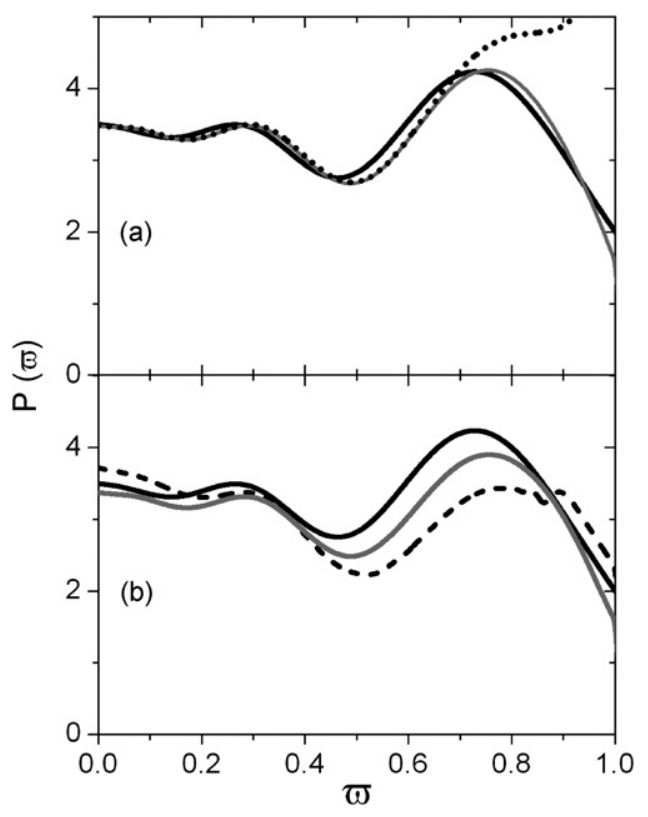

Figure 5. Profiles calculated from equation (42) for $\rho=1.9$ and $\lambda=8$, using (a) $\zeta_{\mp}=\zeta$ : exact and UA (black solid line), UB (grey solid line), CQ (dotted line); (b) $\zeta_{\mp}=\zeta_{\mp}^{l}$ : UAL (dashed line), UBL (grey solid line), compared to the exact result (black solid line).

UAL produces spurious oscillations for $\frac{3}{4} \leqslant \varpi \leqslant 1$, whereas UBL passes smoothly through the region.

Note also the differences in the values of the profile at $\varpi=\varpi_{0}$ obtained for various forms of equation (42). They reflect the corresponding limiting cases, i.e. UB \& UBL $\rightarrow$ $\mathrm{SC}, \mathrm{UAL} \rightarrow \mathrm{EC}$ and $\mathrm{UA} \rightarrow \mathrm{TA}$.

There are further possibilities of constructing the uniform approximation using the local argument. This is accomplished by replacing $\zeta_{\mp}=\zeta$ with $\zeta_{t}$ in the UA expression written either in the form (42) or in the form (30). Since the particular functional dependences on $x_{-}(\varpi), x_{+}(\varpi)$ and $\zeta\left[x_{-}(\varpi), x_{+}(\varpi)\right]$ in these two expressions for $I^{u}(\varpi)$ are not the same, replacing $\zeta\left[x_{-}(\varpi), x_{+}(\varpi)\right]$ with $\zeta_{t}(\varpi)$ yields two different functions of $\varpi$. We label these approximations UAT1 and UAT2, respectively. In both of them the additional information, the third derivative of $f$, is needed in only one point $x=x_{0}$ and not in every Condon point. As might be expected, we obtained very good agreement of UAT1 with the exact and UA profiles. It is so because close to $\varpi_{0}$, one has $\zeta_{t} \approx \zeta$ (see figure 4) and far away from $\varpi_{0}$ the oscillation peak positions are predominantly determined by the true phase difference $f\left(x_{+}\right)-f\left(x_{-}\right)$stemming from the $I_{-}^{q}\left(I_{+}^{q}\right)^{*}$ factor in the interference term. Small deviations appear only for rather small values of the asymptotic parameter, $\lambda=1,2$, and a weakly varying transitional dipole moment, $\rho=0.3$.

On the other hand, in the case of UAT2 the oscillation peak positions are determined by the approximate argument of the $\mathrm{Ai}$ and $\mathrm{Ai}^{\prime}$ functions. We revisit this point in section 4.1.

3.2.4. The Szudy-Baylis approach. In the frequency region where the both RFSPs are real $(0<\varpi<1$ in our case), equation (34) can be written in the form resembling the corresponding coherent quasistatic formula (equations (24) and $\left.\left(26^{\prime}\right)\right)$ :

$$
\begin{aligned}
& P^{u}(\varpi)=\left[P_{-}^{q}(\varpi)+P_{+}^{q}(\varpi)\right] M(\zeta) \\
& \quad+2 \operatorname{sgn}\left[g\left(x_{-}\right) g\left(x_{+}\right)\right] \sqrt{P_{-}^{q}(\varpi) P_{+}^{q}(\varpi)} N(\zeta),
\end{aligned}
$$

where $P_{\mp}^{q}(\varpi)$ are the quasistatic terms given by equation (25) and

$$
\begin{aligned}
M(\zeta) & =\pi\left\{\zeta^{1 / 2}[\mathrm{Ai}(-\zeta)]^{2}+\zeta^{-1 / 2}\left[\mathrm{Ai}^{\prime}(-\zeta)\right]^{2}\right\} \\
N(\zeta) & =\pi\left\{\zeta^{1 / 2}[\mathrm{Ai}(-\zeta)]^{2}-\zeta^{-1 / 2}\left[\operatorname{Ai}^{\prime}(-\zeta)\right]^{2}\right\}
\end{aligned}
$$

The first two terms on the right-hand side of equation (46) can be interpreted as the individual contributions from each of the stationary points, and the third one as due to quantum interference between them [9]. (Our $M(\zeta)$ and $N(\zeta)$ correspond to $\pi P(\zeta)$ and $\pi Q(\zeta)$ of [9]). For $\zeta \gg 1$ one has $M(\zeta) \rightarrow 1$ and $N(\zeta) \rightarrow \sin \left(\frac{4}{3} \zeta^{3 / 2}\right)$, so equation (46) for $P^{u}(\varpi)$ reduces to the CQ expression (24) for $P^{q}(\varpi)$ with $P_{\text {int }}^{q}(\varpi)$ given by equation $\left(26^{\prime}\right)$. Both $M(\zeta)$ and $N(\zeta)$ are singular for $\zeta=0$, and so are also all three terms in equation (46), but their total contribution is finite.

Equation (46) can be rearranged in another way by grouping the contributions comprising either $\mathrm{Ai}$ or $\mathrm{Ai}^{\prime}$ functions:

$$
\begin{aligned}
& P^{u}(\varpi)=\left\{P_{-}^{q}(\varpi)+P_{+}^{q}(\varpi)+2 \operatorname{sgn}\left[g\left(x_{-}\right) g\left(x_{+}\right)\right]\right. \\
& \left.\quad \times \sqrt{P_{-}^{q}(\varpi) P_{+}^{q}(\varpi)}\right\} \pi \zeta^{1 / 2}[\operatorname{Ai}(-\zeta)]^{2} \\
& +\left\{P_{-}^{q}(\varpi)+P_{+}^{q}(\varpi)-2 \operatorname{sgn}\left[g\left(x_{-}\right) g\left(x_{+}\right)\right]\right. \\
& \left.\quad \times \sqrt{P_{-}^{q}(\varpi) P_{+}^{q}(\varpi)}\right\} \pi \zeta^{-1 / 2}\left[\mathrm{Ai}^{\prime}(-\zeta)\right]^{2}
\end{aligned}
$$

To analyze the behaviour of $P^{u}(\varpi)$ in the vicinity of $\varpi_{0}$ (and assuming $\operatorname{sgn} g\left(x_{-}\right)=\operatorname{sgn} g\left(x_{+}\right)$, which is very likely, especially for $x_{ \pm} \rightarrow x_{0}$ ), it is convenient to rewrite equation $\left(46^{\prime}\right)$ as

$$
\begin{aligned}
& P^{u}(\varpi)=\left[P_{-}^{q}(\varpi)+P_{+}^{q}(\varpi)-\eta(\varpi)\right] 2 \pi \zeta^{1 / 2}[\operatorname{Ai}(-\zeta)]^{2} \\
& \quad+\eta(\varpi) 2 \pi \zeta^{-1 / 2}\left[\mathrm{Ai}^{\prime}(-\zeta)\right]^{2}
\end{aligned}
$$

where $\eta(\varpi)=\frac{1}{2}\left(\sqrt{P_{+}^{q}(\varpi)}-\sqrt{P_{-}^{q}(\varpi)}\right)^{2}$. For $\varpi \rightarrow \varpi_{0}$ one has $\eta(\varpi) \rightarrow 0$, which led Szudy and Baylis [8] to neglect both terms with $\eta(\varpi)$ in equation (49) and retain only the term

$$
P_{\mathrm{SB}}^{u}(\varpi)=\left[P_{-}^{q}(\varpi)+P_{+}^{q}(\varpi)\right] 2 \pi \zeta^{1 / 2}[\operatorname{Ai}(-\zeta)]^{2},
$$

which they suggested as a 'simple uniform-type formula'. However, since for $\varpi \rightarrow \varpi_{0}$ both $\eta(\varpi)$ and $\zeta^{1 / 2}(\varpi)$ become small parameters, both $\propto \sqrt{\varpi_{0}-\varpi}$, one has $\eta(\varpi) \zeta^{1 / 2}(\varpi) \propto$ $\varpi_{0}-\varpi \rightarrow 0$ while $\eta(\varpi) \zeta^{-1 / 2}(\varpi) \propto\left(\varpi_{0}-\varpi\right)^{0}$ remains finite and not necessarily small in comparison with $\left[P_{-}^{q}(\varpi)+\right.$ $\left.P_{+}^{q}(\varpi)\right] \zeta^{1 / 2}(\varpi)$. Whether it is negligible or not, i.e. whether $P^{u}(\varpi) \rightarrow P_{\mathrm{SB}}^{u}(\varpi)$, depends on the actual behaviour of the transition dipole moment and the differential potential, as well as on the value of the asymptotic parameter $\lambda$. So, $P^{u}(\varpi) \approx P_{\mathrm{SB}}^{u}(\varpi)$ may be a good approximation for $\varpi \rightarrow \varpi_{0}$ for a slowly varying transition dipole moment $\left(g^{(1)}\left(x_{0}\right) \cong 0\right.$ ) and negligible anharmonicity of the differential potential $\left(f^{(4)}\left(x_{0}\right) \cong 0\right.$ ), as well as for large values of $\lambda$ (irrespective of $g$ and $f$ ), but in general it is not, even for $\varpi \rightarrow \varpi_{0}$. Near 
$\varpi_{0}$ the term $P_{\mathrm{SB}}^{u}(\varpi)$ has the form of the SC approximation, while the full $P^{u}(\varpi)$ reduces to TA. (For the model case, see the discussion after equation (40).)

For $\zeta \gg 1$, the term $P_{\mathrm{SB}}^{u}(\varpi)$ has a wrong asymptotic behaviour since it lacks not only the whole $\mathrm{Ai}^{\prime}$ contribution, but a part of the Ai contribution, $-\eta(\varpi) 2 \pi \zeta^{1 / 2}[\mathrm{Ai}(-\zeta)]^{2}$, as well, as readily seen by comparing equations (49) and (50). However, after randomizing the large phase over a cycle, $P_{\mathrm{SB}}^{u}(\varpi)$ averages out to $P_{-}^{q}(\varpi)+P_{+}^{q}(\varpi)$, the incoherent quasistatic approximation to the spectrum, just as the full $P^{u}(\varpi)$ does. This is readily seen by rewriting equation (49) as

$$
P^{u}(\varpi)=P_{\mathrm{SB}}^{u}(\varpi)-2 \eta(\varpi) N(\zeta)
$$

and noting that for $\zeta \rightarrow \infty$, one has

$$
\begin{aligned}
& \pi \zeta^{\frac{1}{2}}[\operatorname{Ai}(-\zeta)]^{2} \rightarrow \sin ^{2}\left(\frac{4}{3} \zeta^{\frac{3}{2}}+\frac{\pi}{4}\right) \\
& N(\zeta) \rightarrow \sin \left(\frac{2}{3} \zeta^{\frac{3}{2}}\right),
\end{aligned}
$$

which average out to $\frac{1}{2}$ and 0 , respectively. Then, it follows from equations (50) and $\left(46^{\prime \prime}\right)$ that both $P_{\mathrm{SB}}^{u}(\varpi)$ and $P^{u}(\varpi)$ for $\zeta \rightarrow \infty$ average out to $P_{-}^{q}(\varpi)+P_{+}^{q}(\varpi)$.

In the present model case, $P_{\mathrm{SB}}^{u}(\varpi)$ is a rather good approximation to UA within the limited range of parameters, say for $0.3<\rho<0.7,0<\varpi \leqslant 1$ and $\lambda>1$.

Anticipating the need for analytical averaging Szudy and Baylis finally approximate $\zeta(\varpi)$ with either of $\zeta_{\mp}^{l}(\varpi)$,

$$
\begin{gathered}
P_{\mathrm{SB}}^{l}(\varpi)=P_{-}^{q}(\varpi) 2 \pi\left[\zeta_{-}^{l}(\varpi)\right]^{1 / 2}\left\{\mathrm{Ai}\left[-\zeta_{-}^{l}(\varpi)\right]\right\}^{2} \\
+P_{+}^{q}(\varpi) 2 \pi\left[\zeta_{+}^{l}(\varpi)\right]^{1 / 2}\left\{\mathrm{Ai}\left[-\zeta_{+}^{l}(\varpi)\right]\right\}^{2},
\end{gathered}
$$

and it is predominantly this step that causes disagreement with the UA result. We address this issue in section 4.1.

\section{Analytical averaging}

In order to apply the obtained results to the experimental data in gas cell conditions with a temperature $T$, one has to average the spectrum $|a(\Delta \omega)|^{2}$ over the impact parameters $b$ and initial energies $E$ of colliding atoms. Assuming a Maxwellian distribution over energies, the averaged spectrum (the thermally averaged transition rate coefficient) is given by

$$
\begin{aligned}
& W(\Delta \omega, T)=4\left(\frac{2 \pi}{\mu}\right)^{1 / 2}(k T)^{-3 / 2} \int_{E_{\min }}^{\infty} \mathrm{d} E E \\
& \quad \times \exp \left(-\frac{E}{k T}\right) \int_{0}^{b_{\max }(E)} \mathrm{d} b b|a(\Delta \omega ; E, b)|^{2} .
\end{aligned}
$$

The dependence of $a(\Delta \omega ; E, b)$ on $E$ and $b$ in equations (4)(6) is contained in the radial relative velocity

$$
v_{\mathrm{r}}(R ; E, b)=\sqrt{\frac{2}{\mu}\left\{\left[1-\left(\frac{b}{R}\right)^{2}\right] E-V_{\mathrm{cl}}(R)\right\}},
$$

where $V_{\mathrm{cl}}(R)$ is the potential governing the assumed classical trajectory and all energies are measured from $V_{\mathrm{cl}}(\infty)$.

As we are interested in the spectral properties arising mostly from the neighbourhood $\left|R-R_{0}\right| \leqslant 1 / \alpha_{R}$ of the extremum point $R_{0}$ of the differential potential, we approximate $v_{\mathrm{r}}(R ; E, b)$ with its value $v_{\mathrm{r}}\left(R_{0} ; E, b\right)$ in this point. This assumption makes $a(\Delta \omega ; E, b)$ a function of $\Delta \omega$ and $v_{\mathrm{r}}\left(R_{0} ; E, b\right)$, the latter variable comprising its only dependence on $E$ and $b$. This introduces the symmetry

$$
a(\Delta \omega ; E, b)=a\left\{\Delta \omega ;\left[1-\left(b / R_{0}\right)^{2}\right] E, b=0\right\}
$$

and also enables one to change the integration over $b$ into one over the radial component of the kinetic energy at $R_{0}$, $\varepsilon_{\mathrm{r}}\left(R_{0} ; E, b\right)=\left[1-\left(b / R_{0}\right)^{2}\right] E-V_{0}$, with $V_{0}=V_{\mathrm{cl}}\left(R_{0}\right)$, which yields a function of the total kinetic energy $\varepsilon\left(R_{0} ; E\right)=$ $E-V_{0}=\varepsilon_{\mathrm{r}}\left(R_{0} ; E, b=0\right)$, thus reducing the double integral in equation (53) to a single one over $\varepsilon$. Then, an integration by parts over $\varepsilon$ yields

$$
\begin{aligned}
& W(\Delta \omega, T)=2\left(\frac{2 \pi}{\mu k T}\right)^{\frac{1}{2}} R_{0}^{2} \mathrm{e}^{-\frac{V_{0}}{k T}} \int_{E_{\min }-V_{0}}^{\infty} \mathrm{d} \varepsilon \\
& \quad \times \exp \left(-\frac{\varepsilon}{k T}\right)\left|a\left(\Delta \omega ; E=\varepsilon+V_{0}, b=0\right)\right|^{2} .
\end{aligned}
$$

For free states reaching the relevant region $\left|R-R_{0}\right| \leqslant 1 / \alpha_{R}$, the energy $E_{\mathrm{min}}$ equals the maximum value of $V_{\mathrm{cl}}(R)$ between $R=\infty$ and $R_{0}$. Usually, i.e., for $V_{\mathrm{cl}}(R)$ without a maximum in between, $E_{\min }=V_{\mathrm{cl}}(\infty)=0$. However, if one includes the (quasicontinuous) bound states as well, which also do contribute to the process, then $E_{\text {min }} \approx V_{\mathrm{cl}}\left(R_{0}\right)=V_{0}$ and the lower limit of the integral in equation (56) can be replaced by zero.

Introducing the parameter $\bar{\lambda}=\Delta U\left(R_{0}\right) / \hbar \alpha_{R} v_{p}$, where $v_{p}=\sqrt{2 k T / \mu}$ is the most probable asymptotic relative velocity, equation (56) may be finally written as

$$
W(\Delta \omega, T)=C \tilde{W}(\varpi, \bar{\lambda})
$$

where the reduced averaged profile is given by

$$
\tilde{W}(\varpi, \bar{\lambda})=\frac{1}{\sqrt{\pi}} \int_{0}^{\infty} \mathrm{d} x \frac{\mathrm{e}^{-x}}{\sqrt{x}} P\left(\varpi, \lambda=\frac{\bar{\lambda}}{\sqrt{x}}\right)
$$

and

$$
C=8 \pi^{2} \frac{\hbar \gamma_{R}^{2} R_{0}^{2}}{\alpha_{R} \Delta U\left(R_{0}\right)} \mathrm{e}^{-\frac{v_{0}}{k T}} .
$$

For a straight-line trajectory $V_{0}=0$ and the Boltzmann factor $\exp \left(-V_{0} / \mathrm{kT}\right)$ equals unity.

In figure 6 , we compare the exact reduced averaged profiles with the corresponding UA and UAT1 counterparts for four values of the asymptotic parameter, $\bar{\lambda}=1,2,4,8$, and two values of the transition dipole moment parameter, $\rho=0.7,0.3$. For $\rho=0.7$, the differences among the three types of averaged profiles are negligible in the whole region shown. For $\rho=0.3$, the differences are visible only in the vicinity of the line core, where the stationary phase approximation is inapplicable in the first place. From this we conclude that the UA approximation fully describes the spectrum of far wings of spectral lines for frequency shifts outside the Weisskopf limits, i.e. wherever appropriate. The proper treatment of the close vicinity of the line centre requires different approaches and approximations [18]. 


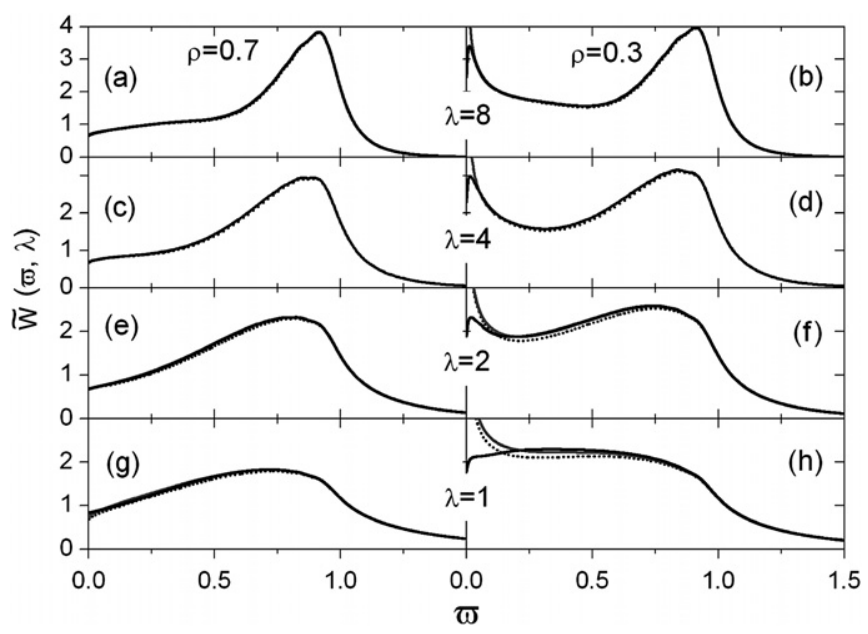

Figure 6. Exact reduced averaged profiles $\tilde{W}(\varpi, \lambda)$ (black solid line) compared with those obtained by the uniform Airy (UA) approximation, $\tilde{W}^{u}(\varpi, \lambda)$, based on the mapping of RFSPs (grey solid line), and the transitional UAT1 approximation (dotted line).

\subsection{Averaged uniform Airy profile}

The reduced averaged profile in the uniform Airy approximation can be written as

$$
\begin{aligned}
& \tilde{W}^{u}(\varpi, \bar{\lambda})=\left[P_{-}^{q}(\varpi)+P_{+}^{q}(\varpi)\right] M_{\mathrm{av}}(z) \\
& \quad+2 \operatorname{sgn}\left[g\left(x_{-}\right) g\left(x_{+}\right)\right] \sqrt{P_{-}^{q}(\varpi) P_{+}^{q}(\varpi)} N_{\mathrm{av}}(z),
\end{aligned}
$$

where

$$
\begin{gathered}
M_{\mathrm{av}}(z)=3 \sqrt{\pi z}\left(L(z)+z^{-1} H(z)\right), \\
N_{\mathrm{av}}(z)=3 \sqrt{\pi z}\left(L(z)-z^{-1} H(z)\right), \\
L(z)=\frac{1}{3} \int_{0}^{\infty} \mathrm{d} x \mathrm{Ai}\left(-z x^{-1 / 3}\right)^{2} x^{-2 / 3} \mathrm{e}^{-x}, \\
H(z)=\frac{1}{3} \int_{0}^{\infty} \mathrm{d} x \mathrm{Ai}^{\prime}\left(-z x^{-1 / 3}\right)^{2} x^{-1 / 3} \mathrm{e}^{-x}, \\
z=\left\{\frac{3}{4} \bar{\lambda}\left[f\left(x_{+}\right)-f\left(x_{-}\right)\right]\right\}^{\frac{2}{3}} .
\end{gathered}
$$

For large positive values $z \gg 1$, one has

$$
3 \sqrt{\pi z} L(z) \rightarrow \frac{1}{2}, \quad 3 \sqrt{\pi / z} H(z) \rightarrow \frac{1}{2}, \quad M_{\mathrm{av}}(z) \rightarrow 1,
$$$$
N_{\mathrm{av}}(z) \rightarrow N_{q}(z),
$$

where

$$
N_{q}(z)=\frac{1}{\sqrt{\pi}} \int_{0}^{\infty} \mathrm{d} x \frac{\mathrm{e}^{-x}}{\sqrt{x}} \sin \left(\frac{4}{3} z^{3 / 2} x^{-1 / 2}\right),
$$

so the reduced averaged profile assumes the coherent quasistatic form

$$
\begin{aligned}
& \tilde{W}^{q}(\varpi, \bar{\lambda})=P_{-}^{q}(\varpi)+P_{+}^{q}(\varpi)+2 \operatorname{sgn}\left[g\left(x_{-}\right) g\left(x_{+}\right)\right] \\
& \quad \times \sqrt{P_{-}^{q}(\varpi) P_{+}^{q}(\varpi)} N_{q}(z) .
\end{aligned}
$$

Both functions $M_{\mathrm{av}}(z)$ and $N_{\mathrm{av}}(z)$ diverge for $z \rightarrow 0$, but the total profile (60) remains finite, as can be seen from the expression for the averaged TA:

$$
\begin{gathered}
\tilde{W}^{t}(\varpi, \bar{\lambda})=6 \sqrt{\pi} g\left(x_{0}\right)^{2} \bar{\lambda}\left(\frac{2}{\bar{\lambda}\left|f^{(3)}\left(x_{0}\right)\right|}\right)^{2 / 3} \\
\times\left[L\left(z_{t}\right)+\left(\frac{g^{(1)}\left(x_{0}\right)}{g\left(x_{0}\right)}-\frac{1}{6} \frac{f^{(4)}\left(x_{0}\right)}{f^{(3)}\left(x_{0}\right)}\right)^{2}\right. \\
\left.\times\left(\frac{2}{\bar{\lambda}\left|f^{(3)}\left(x_{0}\right)\right|}\right)^{2 / 3} H\left(z_{t}\right)\right], \\
z_{t}=\bar{\lambda} f^{(1)}\left(x_{0}, \varpi\right)\left(\frac{2}{\bar{\lambda}\left|f^{(3)}\left(x_{0}\right)\right|}\right)^{1 / 3} .
\end{gathered}
$$

This expression can also be used for analytic continuation into the classically forbidden region, $\varpi>\varpi_{0}$. If the second contribution in the brackets is negligible, the variation of the transition dipole moment and the anharmonicity of the differential potential do not affect the shape of the spectrum.

In our model, the transitional profile has the form

$$
\tilde{W}^{t}(\varpi, \bar{\lambda})=6 \sqrt{\pi}(\bar{\lambda})^{1 / 3}\left[L\left(z_{t}\right)+\left(\rho-\frac{1}{2}\right)^{2}(\bar{\lambda})^{-2 / 3} H\left(z_{t}\right)\right] .
$$

In the special case $\rho=1 / 2$, the contribution of the $\mathrm{Ai}^{\prime}$ term is absent. For $\varpi \approx \varpi_{0}$, this contribution is negligible whenever $(\rho-1 / 2)^{2} \ll 15 \bar{\lambda}^{2 / 3}$, which is considerably less stringent than the analogous condition for a nonaveraged profile, $(\rho-1 / 2)^{2} \ll 1.88 \lambda^{2 / 3}$.

One can say that the model analyzed in this work describes successfully a large realm of realistic physical situations $(0<\rho<1, \bar{\lambda}>1)$ for which the contribution of the second term in the brackets in equation (71) is negligible. However, in the case of optical transitions where the dipole moment $g(x)$ happens to change sign in the vicinity of the extremum of the differential potential [19], it is only the second term of equation (69) that contributes for $\varpi \approx \varpi_{0}$ :

$\tilde{W}^{t}(\varpi, \bar{\lambda})=6 \sqrt{\pi} \bar{\lambda}\left[g^{(1)}\left(x_{0}\right)\right]^{2}\left[\frac{2}{\bar{\lambda}\left|f^{(3)}\left(x_{0}\right)\right|}\right]^{4 / 3} H\left(z_{t}\right)$.

\subsection{Comparison with other theoretical approaches}

At the beginning of section 4 , we assumed that $v_{\mathrm{r}}(R) \approx$ const., the commonest case of which being a straight-line trajectory with zero impact parameter, for which $v_{\mathrm{r}}(R)=v(R)=v(\infty)$. If the trajectory is not a straight line, the Boltzmann factor $\exp \left(-V_{c l}\left(R_{0}\right) / k T\right)$ is different from unity, and $v_{\mathrm{r}}(R) \neq v(\infty)$ (irrespective of $b$ ). In section 3 we treated a special case $v_{\mathrm{r}}(R)=v=$ const. for which there is an analytic solution. For the general case, $v_{r}(R) \neq$ const., all our expressions remain valid provided that one makes the replacements $\lambda[f(x, \varpi)-$ $\left.f\left(x_{\min }, \varpi\right)\right] \rightarrow \tilde{F}\left(x, x_{\min }, \varpi\right)$ and $g(x, \rho) / v \rightarrow \tilde{G}(x, \rho)$, with $\tilde{F}$ and $\tilde{G}$ defined by equations $\left(5^{\prime}\right)$ and $\left(6^{\prime}\right)$. Since $\tilde{F}^{(1)}\left(x, x_{\min }, \varpi\right)=\lambda v f^{(1)}(x, \varpi) / \tilde{v}_{\mathrm{r}}(x)$ and $\tilde{v}_{\mathrm{r}}(x) \neq 0$ for $x \neq x_{\min }$, the phase functions $\tilde{F}$ and $f$ have the same stationary points. 
For the general case, the mapping parameter $\zeta(\varpi)$ can be written as [10]

$$
\begin{aligned}
& \frac{4}{3}[\zeta(\varpi)]^{3 / 2}=\tilde{F}\left[x_{+}(\varpi), x_{-}(\varpi), \varpi\right] \\
& \quad=\frac{\Delta U\left(R_{0}\right)}{\hbar \alpha_{R}} \int_{x_{-}(\varpi)}^{x_{+}(\varpi)} \frac{\mathrm{d} x}{\tilde{v}_{\mathrm{r}}(x)}[\Delta \tilde{U}(x)-\varpi],
\end{aligned}
$$

and defining the parameter $\bar{v}_{\mathrm{r}}$ by the relation

$$
\int_{x_{-}(\varpi)}^{x_{+}(\varpi)} \frac{\mathrm{d} x}{\tilde{v}_{\mathrm{r}}(x)}[\Delta \tilde{U}(x)-\varpi]=\frac{1}{\bar{v}_{\mathrm{r}}} \int_{x_{-}(\varpi)}^{x_{+}(\varpi)} \mathrm{d} x[\Delta \tilde{U}(x)-\varpi],
$$

one has

$$
\left.\frac{4}{3}[\zeta(\varpi)]^{3 / 2}=\frac{\Delta U\left(R_{0}\right)}{\hbar \alpha_{R} \bar{v}_{\mathrm{r}}}\left\{f\left[x_{+}(\varpi), \varpi\right)\right]-f\left[x_{-}(\varpi), \varpi\right]\right\} .
$$

In the final expressions, two local velocities $\tilde{v}_{\mathrm{r}}\left(x_{ \pm}\right)$appear in the contributions due to two stationary points, as well as the above-defined average velocity $\bar{v}_{\mathrm{r}}$. In particular, $P_{ \pm}^{q}=\left[v / \tilde{v}_{\mathrm{r}}\left(x_{ \pm}\right)\right]\left|g\left(x_{ \pm}\right)\right|^{2} /\left|f^{(2)}\left(x_{ \pm}\right)\right|$. For the averaging to be analytical, one has to have a single local velocity in each of the terms in equation (60), which calls for certain approximations. Beuc and Horvatic [10] chose the following ones: they used equation (75) with $\bar{v}_{\mathrm{r}} \approx \tilde{v}_{\mathrm{r}}\left(x_{ \pm}\right)$for the arguments of $M(\zeta), \bar{v}_{\mathrm{r}} \approx \tilde{v}_{\mathrm{r}}\left(x_{-}\right)$for the argument of $N(\zeta)$, and $\sqrt{\tilde{v}_{\mathrm{r}}\left(x_{-}\right) \tilde{v}_{\mathrm{r}}\left(x_{+}\right)} \approx \tilde{v}_{\mathrm{r}}\left(x_{-}\right)$in the product $P_{-}^{q}(\varpi) P_{+}^{q}(\varpi)$ to 'decouple' all three terms in equation (60). Vicharelli and Collins [9] used instead the local approximations $\zeta_{ \pm}^{l}$ in the $M$ functions but $\bar{\zeta}=\left(\zeta_{-}^{l} \zeta_{+}^{l}\right)^{1 / 2}$ for the argument of $N(\zeta)$ and in the product $P_{-}^{q}(\varpi) P_{+}^{q}(\varpi)$. Note that in our model case assuming a straight-line trajectory and zero impact parameter the BeucHorvatić approach leads to the exact mapping parameter $\zeta$, while in the Vicharelli-Collins approach the approximate local values $\zeta_{ \pm}^{l}$ and $\bar{\zeta}$ remain approximate.

The final formula obtained by Beuc and Horvatić [10] is identical in form to the Vicharelli-Collins formula [9], but differs in the definitions of arguments used.

Equation (60) as well as its analytic continuation into the antistatic region via equation (69) represents the same profile as proposed by Beuc and Horvatić, with the only difference that they did not take into account the contribution of the anharmonicity of the differential potential, $\propto f^{(4)}\left(x_{0}\right) / f^{(3)}\left(x_{0}\right)$.

The averaging of the relation $\left(46^{\prime \prime}\right)$ yields the averaged profile in the form

$$
\tilde{W}^{u}(\varpi, \bar{\lambda})=\left[P_{-}^{q}(\varpi)+P_{+}^{q}(\varpi)\right] 6 \sqrt{\pi z} L(z)-R_{\mathrm{av}}(z),
$$

where

$R_{\mathrm{av}}(z)=\left\{\sqrt{P_{-}^{q}(\varpi)}-\operatorname{sgn}\left[g\left(x_{-}\right) g\left(x_{+}\right)\right] \sqrt{P_{+}^{q}(\varpi)}\right\}^{2} N_{\mathrm{av}}(z)$.

As $N_{\mathrm{av}}(z)$ decays quickly on both sides of the extremum, exponentially for $z<0$ and oscillatorily for $z>0$, it suffices to know the function $R_{\mathrm{av}}(z)$ around the extremum.

If the contribution of $R_{\mathrm{av}}(z)$ is negligible, the spectral profile is given by the simple formula

$$
\tilde{W}^{u}(\varpi) \approx\left[P_{-}^{q}(\varpi)+P_{+}^{q}(\varpi)\right] 6 \sqrt{\pi z} L(z) \equiv \tilde{W}_{\mathrm{SB}}^{u}(\varpi),
$$

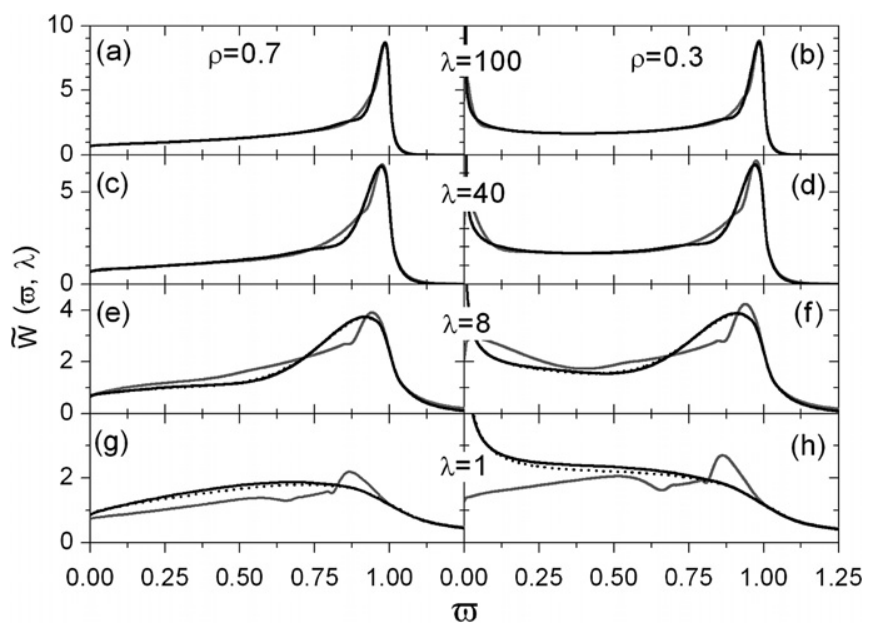

Figure 7. Comparison of the UA (black solid line) and RUAT2 (black dotted line) reduced averaged profiles with the Szudy-Baylis one (grey solid line) for the asymptotic parameter values $\lambda=1,8,40,100$ and $\rho=0.7$ (left column) and $\rho=0.3$ (right column).

which is the averaged $P_{\mathrm{SB}}^{u}(\varpi)$, equation (50). We name $\tilde{W}_{\mathrm{SB}}^{u}(\varpi)$ the reduced uniform Airy (RUA) approximation.

If in $\tilde{W}_{\mathrm{SB}}^{u}(\varpi)$ one replaces $z$ with its transitional form $z_{t}$, what one obtains is the averaged UAT2 approximation with $R_{\mathrm{av}}\left(z_{t}\right)=0$, which we name RUAT2, the reduced (averaged) UAT2. (UAT2 is given by equation $\left(46^{\prime}\right)$ or (49) with $\zeta$ replaced by $\zeta_{t}$, the averaged UAT2 by equation (60) or (76) with $z$ replaced by $z_{t}$, and RUAT2 is then obtained by neglecting $R_{\mathrm{av}}\left(z_{t}\right)$.) In both cases the term 'reduced' refers to neglecting the term $R_{\mathrm{av}}(z)$ or $R_{\mathrm{av}}\left(z_{t}\right)$, respectively, not to the averaged transition rate coefficient $W$ being 'reduced' to the dimensionless quantity $\tilde{W}=W / C$.

Introducing local variables, one obtains

$\tilde{W}_{\mathrm{SB}}^{l}(\varpi)=P_{-}^{q}(\varpi) 6 \sqrt{\pi z_{-}} L\left(z_{-}\right)+P_{+}^{q}(\varpi) 6 \sqrt{\pi z_{+}} L\left(z_{+}\right)$,

which is the averaged $P_{\mathrm{SB}}^{l}(\varpi)$, given by equation (52), and is identical to the relation proposed by Szudy and Baylis [8].

For $z \gg 1$, one has $6 \sqrt{\pi z} L(z) \rightarrow 1$, so the spectral profile $\tilde{W}_{\mathrm{SB}}^{u}(\varpi)$ reduces to the incoherent quasistatic one,

$$
\tilde{W}_{\mathrm{SB}}^{u}(\varpi) \rightarrow P_{-}^{q}(\varpi)+P_{+}^{q}(\varpi),
$$

and it is for this reason that this type of approximation has been inappropriately saddled with the name 'non-coherent uniform Airy approximation' [10]. In fact $P_{\mathrm{SB}}^{u}(\varpi)$ and $\tilde{W}_{\mathrm{SB}}^{u}(\varpi)$ do comprise a part of the interference term proportional to $\zeta^{1 / 2}[\mathrm{Ai}(-\zeta)]^{2}$ and $\sqrt{z} L(z)$, respectively, to the extent that $\left(P_{-}^{q}+P_{+}^{q}\right) / 2 \approx \sqrt{P_{-}^{q} P_{+}^{q}}$. For $P_{-}^{q} \rightarrow P_{+}^{q}$, i.e. for $\varpi \rightarrow \varpi_{0}$ they comprise all of it, and it constitutes one half of the total expression.

The comparison of the Szudy-Baylis profile, obtained from equation (79), with the averaged UA one in figure 7 shows considerable differences, especially in the region around the inflection point of the differential potential. The maximum is also considerably sharper than that in the UA profile, which is a fact that had been noticed in the comparison with experimental 
spectra as well. These differences vanish only for extremely large values of the asymptotic parameter, $\lambda>40$.

On the other hand, the averaged RUAT2 profile fares mисh better, showing slight differences with respect to the UA profile only for the smallest value of $\lambda$ shown, $\lambda=1$. We conclude that the averaged RUAT2 represents a numerically quite satisfactory approximation in a large range of parameters. It can be said to unite two pioneering works in the field of satellite rainbow theory, with its form following the expression proposed by Szudy and Baylis and the argument of the Airy function being of a transitional form as proposed by Sando and Wormhoudt.

The above considerations show that the reason for the discrepancy of the Szudy-Baylis results with experiment lies not in the omission of the function $\zeta^{-1 / 2}\left[\mathrm{Ai}^{\prime}(-\zeta)\right]^{2}$, or the alleged 'incoherence', but in the choice of $\zeta(\varpi)$ as obtained by the local approximation.

\section{Conclusions}

The analytical model proposed by Devdariani et al in which the Morse potential is used to model the difference potential and the optical transition probability (or radiation width) is approximated by the exponential function, lends itself to the analysis of a wide class of optical transitions where the difference potential has a single extremum and the transition dipole moment varies monotonously in the vicinity of this extremum. Within this model, under the additional assumption of a constant radial relative velocity, the optical transition spectrum for a given collision energy and impact parameter can be calculated analytically. These exact spectra were compared to those obtained by various asymptotic methods based on the stationary phase approximation, which are also obtained in the closed (analytic) form. In this way, we could estimate the applicability and accuracy of these approximate procedures.

Already the simplest, coherent quadratic approximation shows good agreement with the exact profile for frequencies outside the region of the 'line core', bounded by the Weisskopf limits, as well as far enough from the classical singularity, i.e., in the quasistatic part of the spectrum proper. In this frequency region practically the same results are obtained whether one sums the leading asymptotic contributions of the real-frequency stationary points (which are also real in this region), or one includes the time dependence of the dipole moment into the phase function, which makes both the frequency and the stationary points complex.

The interference of the contributions of different stationary points has a profound influence on the shape of the spectrum, which points to the importance of the accuracy of the phase difference between the stationary points.

The Airy approximation based on the uniform mapping gives excellent agreement with the exact profile in the whole frequency region except in the vicinity of the line centre, which is the proper realm of the impact line broadening theory. The procedure based on the uniform mapping with real-frequency stationary points shows somewhat better agreement than the one with complex-frequency points in the whole range of its applicability. Also, for the calculation of spectra in a real physical situation, it is numerically simpler to work out the real stationary points than the complex ones. So, one of the most important results of this work is the confirmation of the validity of the uniform Airy approximation based on the mapping with real-frequency stationary points.

In the vicinity of the classical singularity the uniform Airy approximation assumes a simpler form termed the transitional approximation. We established that the spectral intensity in this region depends not only on the value and rate of change of the transition dipole moment, but also on the degree of anharmonicity of the differential potential in the vicinity of the extremum.

The Airy approximation based on the local approximation of the phase difference shows substantial deviations from the exact values despite being of the same form as the uniform Airy approximation. The reason for the discrepancy lies in the inaccuracy of the argument of the Airy functions which does not take into account the actual phase difference of the stationary points.

We also analyzed the spectra averaged over the statistical ensemble, i.e. over the initial energies and impact parameters of colliding atoms. We found almost complete agreement of the averaged exact spectrum with that obtained by the uniform Airy approximation. The approximation of the argument of Airy functions with its transitional form, UAT2, also gives very good results, which are barely distinguishable from the exact ones only for small values of the asymptotic parameter, $\bar{\lambda} \approx 1$.

The uniform Airy approximation was also reduced to a simplified expression (RUA) which has the same form as the well-known relation proposed by Szudy and Baylis and is very convenient for practical application in real physical situations. We showed that the main defect of the SzudyBaylis approach is not due to the alleged 'incoherence', but to the choice of the argument of Airy functions as obtained by the local approximation.

Bieniek et al [20] have analyzed analogous processes of ionizing collisions in complex potentials within uniform JWKB stationary-phase techniques. Their numerical results are in excellent agreement with fully quantal, complexpotential computations. Our new results additionally support the conclusions reached in [10] and [20] that the UA approach is suitable for the description of a broad class of asymptotically forbidden but collisionally induced transitions.

\section{Acknowledgment}

We acknowledge financial support from the Ministry of Science, Education, and Sports of the Republic of Croatia, Project No 035-0352851-3213.

\section{References}

[1] Berman P R, Schuller F and Nienhuis G 1990 Phys. Rev. A 42459

[2] Szudy J and Baylis W E 1996 Phys. Rep. 266127

[3] Sando K M and Wormhoudt J C 1973 Phys. Rev. A 71889

[4] Devdariani A 1979 Opt. Spectrosc. 4758 
[5] Devdariani A, Bichoutskaia E, Tchesnokov E, Bichoutskaia T, Crothers D S F, Leboucher-Dalimier E, Sauvan P and Angelo P 2002 J. Phys. B: At. Mol. Opt. Phys. 352469

[6] Connor J N L and Marcus R A 1971 J. Chem. Phys. 555636

[7] Connor J N L 1973 Mol. Phys. 25181

[8] Szudy J and Baylis W E 1975 J. Quant. Spectrosc. Radiat. Transfer 15641

[9] Vicharelli P A and Collins C B 1983 Spectral Line Shapes vol 2 ed K Burnett (Berlin: de Gruyter) p 537

[10] Beuc R and Horvatic V 1992 J. Phys. B: At. Mol. Opt. Phys. 251497

[11] Abramowitz M and Stegun I A 1964 Handbook of Mathematical Functions with Formulas, Graphs, and
Mathematical Tables (National Bureau of Standards Applied Mathematics Series Number 55) (Washington, DC: US Govt Printing Office)

[12] Bleistein N and Handelsman R A 1986 Asymptotic Expansions of Integrals (New York: Dover)

[13] Berry M V 1989 Proc. R. Soc. Lond. A 4227

[14] Miller W H 1970 J. Chem. Phys. 533578

[15] Bieniek R J 1976 Chem. Phys. Lett. 4072

[16] Walkup R E 1982 Phys. Rev. A 25596

[17] Bieniek R J and Streeter T J 1983 Phys. Rev. A 283328

[18] Gallagher A and Holstein T 1977 Phys. Rev. A 162413

[19] Devdariani A and Dalimier E 2008 Phys. Rev. A 78022512

[20] Bieniek R J, Müller M W and Movre M 1990 J. Phys. B: At. Mol. Opt. Phys. 234521 